\title{
Effectiveness and Economic Evaluation of Chiropractic Care for the Treatment of Low Back Pain: A Systematic Review of Pragmatic Studies
}

\author{
Marc-André Blanchette ${ }^{1}$, Mette Jensen Stochkendahl ${ }^{2}$, Roxane Borges Da Silva ${ }^{3}$, \\ Jill Boruff ${ }^{4}$, Pamela Harrison ${ }^{4}$, André Bussières ${ }^{4,5,6}$ \\ 1 Public Health PhD Program, School of Public Health, University of Montreal, Montreal, QC, Canada, \\ 2 Nordic Institute of Chiropractic and Clinical Biomechanics, Odense, Denmark, 3 Faculty of Nursing, \\ University of Montreal, Montreal, QC, Canada, 4 School of Physical and Occupational Therapy, Faculty of \\ Medicine, McGill University, Montreal, QC, Canada, 5 Centre de Recherche Interdisciplinaire en \\ Réadaptation de Montréal, Montreal, QC, Canada, 6 Département chiropratique, Université du Québec à \\ Trois-Rivières, Trois-Rivières, QC, Canada \\ * marc-andre.blanchette@ @umontreal.ca
}

\section{f open access}

Citation: Blanchette M-A, Stochkendahl MJ, Borges Da Silva R, Boruff J, Harrison P, Bussières A (2016) Effectiveness and Economic Evaluation of Chiropractic Care for the Treatment of Low Back Pain: A Systematic Review of Pragmatic Studies. PLOS ONE 11(8): e0160037. doi:10.1371/journal. pone. 0160037

Editor: Tim Friede, University Medical Center Göttingen, GERMANY

Received: April 26, 2016

Accepted: July 12, 2016

Published: August 3, 2016

Copyright: @ 2016 Blanchette et al. This is an open access article distributed under the terms of the Creative Commons Attribution License, which permits unrestricted use, distribution, and reproduction in any medium, provided the original author and source are credited.

Data Availability Statement: All relevant data are within the paper and its Supporting Information files.

Funding: The authors received no specific funding for this work. Participation in this work is paid by in kind contributions from the authors' respective funders and institutions. The publication fees were paid by the lead author's PhD fellowship granted by the Canadian Institute of Health Research. Dr. Stochkendahl's position is funded by the Danish Chiropractic Research Foundation. Dr. Bussieres's position at McGill University is funded by the

\section{Abstract}

\section{Background Context}

Low back pain (LBP) is one of the leading causes of disability worldwide and among the most common reasons for seeking primary sector care. Chiropractors, physical therapists and general practitioners are among those providers that treat LBP patients, but there is only limited evidence regarding the effectiveness and economic evaluation of care offered by these provider groups.

\section{Purpose}

To estimate the clinical effectiveness and to systematically review the literature of full economic evaluation of chiropractic care compared to other commonly used care approaches among adult patients with non-specific LBP.

\section{Study Design}

Systematic reviews of interventions and economic evaluations.

\section{Methods}

A comprehensive search strategy was conducted to identify 1) pragmatic randomized controlled trials (RCTs) and/or 2) full economic evaluations of chiropractic care for low back pain compared to standard care delivered by other healthcare providers. Studies published between 1990 and $4^{\text {th }}$ June 2015 were considered. Primary outcomes included pain, functional status and global improvement. Study selection, critical quality appraisal and data extraction were conducted by two independent reviewers. Data from RCTs with low risk of 
Canadian Chiropractic Research Foundation. The funders had no role in study design, data collection and analysis, decision to publish, or preparation of the manuscript.

Competing Interests: The authors have read the journal's policy and the authors of this manuscript have the following competing interests: MAB:

Personal fees from Ordre des chiropraticiens du Québec for one teaching presentation, outside the submitted work. MJS: Position at the Nordic Institute of Chiropractic and Clinical Biomechanics is funded by the Danish Chiropractic Research Foundation. The Foundation had no role in the study design; in the design and conduct of the study, in the collection, management, analysis, and interpretation of data; in the preparation, review or approval of the manuscript; or in the decision to submit the article for publication. RBDS: Nothing to disclose. JB: Nothing to disclose. PH: Nothing to disclose. AB: Position at the School of Physical and Occupational Therapy at McGill University is funded by the Canadian Chiropractic Research Foundation. The Foundation had no role in the study design; in the design and conduct of the study, in the collection, management, analysis, and interpretation of data; in the preparation, review or approval of the manuscript; or in the decision to submit the article for publication.

Abbreviations: AMD, Adjusted Mean Difference; BMJ, British Medical Journal; CADTH, Canadian Agency for Drugs and Technologies in Health; $\mathrm{ClHI}$, Canadian Institute for Health Information; ClHR, Canadian Institute of Health Research; CPGs, Clinical Practice Guidelines; CPT, Current Procedural Terminology; DC, Doctor of Chiropractic; DCPm, Chiropractic care with physical modalities; GDPD, Gross Domestic Product Deflator; HMO, Health Maintenance Organization; ICL, Index to Chiropractic Literature; LBP, Low Back Pain; MD, Medical Doctor; MD/DO, Medical Doctor or Doctor of Osteopathy; MDPt, Medical Doctor and Physical therapy; NICE, National Institute for Health and Care Excellence; PPP, Purchasing Power Parities; QALYs, Quality Adjusted Life Years; RCTs, Randomized controlled trial; SD, Standard Deviation; SMT, Spinal Manipulative Therapy; Sx/ED, Surgeons and Emergency Department; US, United-States; USD, United-States Dollard; UK, United Kingdom; VAS, Visual Analogue Scale. bias were included in a meta-analysis to determine effect estimates. Cost estimates of full economic evaluations were converted to 2015 USD and results summarized using Slavin's qualitative best-evidence synthesis.

\section{Results}

Six RCTs and three full economic evaluations were scientifically admissible. Five RCTs with low risk of bias compared chiropractic care to exercise therapy $(n=1)$, physical therapy $(n=3)$ and medical care $(n=1)$. Overall, we found similar effects for chiropractic care and the other types of care and no reports of serious adverse events. Three low to high quality full economic evaluations studies (one cost-effectiveness, one cost-minimization and one cost-benefit) compared chiropractic to medical care. Given the divergent conclusions (favours chiropractic, favours medical care, equivalent options), mixed-evidence was found for economic evaluations of chiropractic care compared to medical care.

\section{Conclusion}

Moderate evidence suggests that chiropractic care for LBP appears to be equally effective as physical therapy. Limited evidence suggests the same conclusion when chiropractic care is compared to exercise therapy and medical care although no firm conclusion can be reached at this time. No serious adverse events were reported for any type of care. Our review was also unable to clarify whether chiropractic or medical care is more cost-effective. Given the limited available evidence, the decision to seek or to refer patients for chiropractic care should be based on patient preference and values. Future studies are likely to have an important impact on our estimates as these were based on only a few admissible studies.

\section{Background}

Low back pain (LBP) is the most common occupational disorder in North America [1, 2], a major cause of work absenteeism [3,4] and a leading cause of disability worldwide [5]. The 2010 Global Burden of Disease Study revealed that the LBP disability-adjusted life years increased from 58.2 million in 1990 to 83.0 million in 2010[6], although the majority of LBP patients experience non-specific symptoms that cannot be attributed to a serious disease [7]. The global point prevalence of LBP is 9.4\% [6] and the life time prevalence is around $85 \%$ [8, 9].

A number of factors drive patients' choice for a specific health provider. First, access to professionals is influenced by a traditional medical model of referral [10]. Other structural factors include the regional supply of providers $[11,12]$ or coverage of provider services in medical insurance schemes [13]. Financial reasons (such as out of pocket expenses), socio-cultural (such as traditions) and personal beliefs and preferences are also driving factors [14]. Perhaps as a result, patients with LBP tend to first consult general physicians [10]. However, an increasing number of LBP sufferers seek care directly from other healthcare professionals $[15,16]$. From 2006 to 2010, the proportion of patients self-referring to physiotherapists in The Netherlands rose from $22 \%$ to $43 \%$ [17]. LBP patients also commonly seek chiropractic care [18-20]. At least one third of back pain patients in Denmark choose to see a chiropractor as their entry into the healthcare system [15]. 
North American health technology assessments on chiropractic care conducted over a decade ago were unable to provide clear guidance to inform decision-making on the effectiveness and cost effectiveness of chiropractic care compared to medical and physiotherapy care $[21,22]$. This was primarily because their analysis was based on a limited number of studies of acceptable methodological quality and partial economic evaluations (cost description, cost analysis and cost-outcome description) [21,22].

In a fastidious trial, the efficacy of spinal manipulative therapy (SMT) is tested under optimal conditions in order to isolate its effect from the confounding factors [23]. While in a pragmatic trial, the effectiveness of chiropractic care is tested in close to 'real-world' clinical settings to measure the degree of beneficial effect under this type of conditions [23]. More recently, systematic reviews on the effectiveness of SMT combined the two types of studies and concluded that this approach was as effective as other commonly used treatment modalities [24-26]. While SMT is an important component of chiropractic care [27], these providers commonly use multimodal care to treat their patients with LBP in order to enhance treatment outcome [28] [29]. SMT is also performed by a range of other healthcare professionals [25, 26]. Thus, studies evaluating the effectiveness of SMT can guide clinicians in the choice of treatment modality, but provide little guidance to patients regarding which healthcare provider they should seek care from.

When referring to other providers, inter-professional relations appear to be important to clinicians, where cost is likely a major driver for third party payers when deciding to include a type of provider within a healthcare plan [30-32]. However, healthcare providers, policy makers and third party payers should likely consider the clinical effectiveness, the cost-effectiveness, the safety of the approach, and patient preference and values when referring (LBP) patients for a specific type of care or including a service in a medical insurance scheme, [24, 25, 33]. Full economic evaluation (cost-effectiveness analysis, cost-utility analysis, cost-benefit analysis) of standard care practice offers the advantage of simultaneously considering the resources involved (costs) and the health outcomes (outputs) [34]. This is important when formulating recommendations on the optimal use of healthcare resources [35]. This review therefore aimed to synthetize recent high quality evidence to better inform patients, clinicians, policy makers, and third party payers about the clinical effectiveness and cost-effectiveness of standard chiropractic care for LBP in comparison to usual standard care provided by other healthcare providers. The specific objectives of this review were: 1) to estimate the extent to which chiropractic care is effective for adult patients with non-specific low back pain compared to other conservative care approaches (e.g. medical care and physiotherapy), and 2) to systematically review the literature of full economic evaluation of chiropractic care for adult patients with non-specific LBP compared with other conservative care approaches conducted from any perspective.

\section{Materials and Methods}

\section{Eligibility criteria}

We conducted two systematic reviews: 1) a review of clinical effectiveness and 2) an economic review.

Study characteristics. For the clinical effectiveness review, only randomised controlled trials were eligible for inclusion. For the economic review, studies with a full economic evaluation (i.e. cost-effectiveness, cost-utility, cost-benefit analyses and cost-minimization analysis alongside a clinical trial [36]) were eligible.

Population under study. Adult patients ( $\geq 18$ years) with non-specific LBP with or without sciatica of any duration were eligible for inclusion. We excluded studies reporting on spinal 
pain without separate results for LBP and studies examining specific pathologies (e.g., disc herniation or compression fractures).

Types of interventions. We compared pragmatic trials of chiropractic standard care with standard care delivered by other healthcare providers. Chiropractic standard care was defined as patient-centred, multimodal care (e.g. combinations of SMT, soft tissue techniques, prescription of exercise, advice and reassurance) planned and delivered by a licensed chiropractor. We excluded studies investigating chiropractic care combined with care delivered by other healthcare providers, studies investigating a specific treatment modality or technique, and trials aiming to isolate the effect of SMT (i.e. fastidious trials). The lead authors of potentially relevant studies were contacted for further clarity regarding type of care.

The comparators were conservative, standard care for LBP delivered by other healthcare providers (e.g. medical doctor, physical therapist, exercise therapist or acupuncturist). Studies were judged eligible if the original author, when contacted for clarification, considered the comparator group as standard care. Study arms including surgical treatment or multidisciplinary care were excluded.

\section{Types of outcome measures}

- For the clinical effectiveness review:

Primary outcomes:

- Pain (e.g., visual analogue scale, numerical rating scale, McGill pain score)

- Functional status (e.g. Roland-Morris questionnaire, Oswestry Disability Index)

- Global improvement (e.g., the proportion of patients recovered)

Secondary outcomes:

- Health related quality of life (e.g., SF-36, EuroQol)

- Return to work (e.g. number of days to return to work or proportion of patients at work)

- Adverse events

- For the economic review: an incremental measure of the extra cost required to improve an additional unit of outcome (e.g., an incremental cost-effectiveness ratio (ICER) or an incremental net benefit measure) with the exception of cost-minimization studies for which only costs were considered.

Additional criteria. Studies published in languages other than English or French, duplicate publications and studies without full text manuscript available (e.g. abstracts, conference proceedings, presentations) were excluded.

\section{Information sources}

Electronic searches. We developed our search strategies with an experienced health sciences librarian (JB) (The complete search strategies can be found in the published protocol [37]). A second librarian (PH) reviewed the search strategy for completeness and accuracy. We searched the following databases: Ovid Medline, Ovid AMED, Ovid EMBASE, CINAHL, the Cochrane Database of Systematic Reviews, and PubMed. We further searched for economic evaluations in four additional databases: Index to Chiropractic Literature (ICL); Cochrane Library; Health Technology Assessment Database; and ECONLIT. We searched all bibliographic databases from 1990 to 7th June 2016. 
The search strategies (clinical effectiveness and economic evaluation) were first developed in MEDLINE and subsequently adapted to the other bibliographic databases. The search terms included subject headings (eg, MeSH) specific to each database and free-text words relevant to low back pain. We used Endnote (version X7.3.1, Thomson Reuters, Philadelphia, PA, USA) to create a bibliographic database to manage the search results.

Other resources. We screened reference lists of relevant publications, including reviews and meta-analyses, for relevant articles, and reviewed the gray literature available from the following websites: Canadian Institute for Health Information (CIHI); Canadian Agency for Drugs and Technologies in Health (CADTH); Canadian Institute of Health Research (CIHR); Tufts Medical Center Cost-effectiveness Analysis Registry; Agency for Healthcare Research and Quality; National Institute for Health Research Health Technology Assessment program; and National Institute for Health and Care Excellence (NICE).

\section{Study selection}

Two pairs of authors independently screened titles, abstracts, and full text papers for the clinical effectiveness (MAB and MJS) and for the economic evaluation reviews (MAB and $\mathrm{AB}$ ). $\mathrm{A}$ third reviewer (MJS) was included in the full text screening of the economic evaluations. Reviewers met to resolve disagreements and reach consensus on the eligibility of studies.

\section{Quality assessment}

Clinical effectiveness studies. Two reviewers with expertise related to chiropractic care and clinical trials (MAB and MJS) critically appraised the internal validity of included studies using the 13 criteria recommended by the Cochrane Back Review Group [38]. Studies that met at least 6 criteria out of 13 were considered low risk of bias. Items related to the blinding of patients, care providers, and outcome assessors (patient reported outcomes) were reported in the risk of bias assessment, but cannot be fulfilled when studying the outcome of different groups of healthcare providers.

Economic evaluations. The methodological quality of the economic evaluation was independently assessed by two reviewers using the Drummond BMJ Check list [39-41]: one with expertise related to chiropractic care and epidemiology (MAB) and one with expertise related to healthcare administration and health economics (RBDS). The checklist included 35 items related to study design; data collection; data analysis; and interpretation of results. Additionally, the reviewers formulated a qualitative appreciation of the quality level (low, medium, high) of every study:

High quality: The majority of the quality assessment criteria are met. There is little or no methodological flaw that might influence the study conclusion.

Medium quality: Most of the quality assessment criteria are met. Some flaws in the study may influence the study conclusion.

Low quality: Either most of the quality assessment criteria are not met, or significant flaws relating to key aspects of study are likely to influence the study conclusion.

\section{Data extraction}

Data extraction was performed by the lead investigator (MAB) and checked for accuracy against the original publication by a second reviewer for the clinical effectiveness trial (MJS) and the economic evaluation (RBDS).

The standard form developed by the Cochrane back review group [42] was used to extract descriptive and outcome data from the clinical effectiveness studies. Authors of studies only reporting between group differences [43-46] were unsuccessfully contacted in order to obtain 
mean and standard deviation of primary outcomes at relevant time points. Therefore, the means were inferred from graphical representations and our analysis were based on the reported between group differences. Data extracted from studies at high risk of bias were not reported.

A customized data extraction form [37] was used for the economic evaluations. Authors of the economic evaluations were contacted in order to gain precision about the perspective of analysis and the type of care considered.

\section{Data analysis}

Clinical effectiveness studies. Primary and secondary outcomes of studies with low risk of bias were evaluated in meta-analyses. Final scores of reported outcomes were used for the metaanalyses. Data were estimated, using the Review Manager calculator (RevMan version 5.3, The Cochrane Collaboration, Copenhagen, Denmark) when change scores and between group differences were reported. Outcomes were assessed at 1,3 , and 12 months. In order to minimize clinical diversity, we stratified by: healthcare provider (chiropractic care versus medical care, physical therapy and exercise therapy), symptom duration (sub-acute/chronic (6 weeks or more) and $\mathrm{mix} /$ not specified), and outcomes (type of outcome and time of assessment). To facilitate comparison between different instruments, comparisons were made using standardized mean difference for continuous outcomes and a risk ratio for dichotomous outcomes. Due to substantial heterogeneity, we used a random effect model. Heterogeneity was investigated by subjective interpretation and by statistical testing using the $\mathrm{Q}\left(\mathrm{Chi}^{2}\right)$, $\mathrm{Tau}^{2}$ and $\mathrm{I}^{2}$ test. We, a priori, determined a cut-off of $40 \%$ at the $\mathrm{I}^{2}$ test for reporting pooled estimates. However, due to the limited number of studies, we decided to present the pooled results along with potential sources of heterogeneity even if this criterion was not met. Sensitivity analysis by adding studies with high risk of bias and construction of funnel plots to evaluate possible publication bias were planned [37], but not conducted because the high risk of bias study retrieved only reported results graphically and the number of studies retrieves was too low to construct a meaningful funnel plot. All metaanalyses calculations and related metrics were conducted in Review Manager for Mac OS X (RevMan version 5.3, The Cochrane Collaboration, Copenhagen, Denmark).

Economic evaluations. In order to compare costs across different currencies and account for differential timing, costs of the original study were converted to 2015 United-States (US) dollar using a web-based tool based on purchasing power parities (PPP) and gross domestic product deflators (GDPD) values from the World Economic Outlook Database [47]. The level of evidence of the economic findings was assessed using a 5-point ordinal scale defined by Slavin's qualitative best-evidence synthesis approach [48]. The following criteria were assessed in stepwise descending manner:

Strong Evidence: minimum of 3 high quality studies; at least three-quarters of high and medium quality studies must concur on findings.

Moderate Evidence: minimum of 2 high quality studies, or 3 of medium and high quality; more than two thirds of all studies must report consistent findings.

Limited Evidence: minimum of 1 high quality study or 2 medium quality studies, more than $50 \%$ of all studies must report consistent findings.

Mixed Evidence: findings from medium and high quality studies are contradictory.

Insufficient/No Evidence: no high quality studies; one or no medium quality studies; any number of low quality studies.

\section{Reporting}

The systematic review was organized and reported based on the Preferred Reporting Items for Systematic Reviews and Meta-Analyses statement[49]. 


\section{Systematic review registration}

The protocol was registered with the International Prospective Register of Systematic Reviews on October 20, 2014 (CRD42014008746) at: http://www.crd.york.ac.uk/PROSPERO.

FDA device/drug status: Not applicable.

\section{Results \\ Clinical effectiveness}

Study selection. Our search retrieved 4,095 articles. We removed 1,764 duplicates and screened 2,331 articles for eligibility (Fig 1). After screening, 2,281 articles did not meet our selection criteria, whereas 50 citations were assessed for eligibility in full-text. Eight citations [43-46, 50-53] originating from six individual studies were included in the review (Fig 1). The main reasons for exclusion after full test screening were: care not provided by a chiropractor or investigation of a specific treatment modality. One potentially relevant study with no results available was identified on clinicaltrials.gov (NCT01357343).

Study characteristics. Characteristics of the included studies are presented in Table 1. One Canadian study [52] comparing chiropractic care to physical therapy care had a high risk of bias; therefore we did not include its results into our quantitative analysis and did not further report study findings. The remaining five low risk of bias studies were published between 1990 and 2011 and were conducted in the US $(\mathrm{n}=3)$ [45, 46, 50-52], United Kingdom $(\mathrm{UK}, \mathrm{n}=1)$ $[43,44]$, and Denmark $(n=1)$ [53]. Chiropractic care was compared to exercise therapy care in one study [50], to physical therapy care in four studies $[43,44,51,53]$ and to medical care in one study $[45,46]$. Cohort sizes varied from 155-741 participants and follow-up periods varied from one to three years. We obtained effect estimates for all our primary and secondary outcomes with the exception of return to work. Only the two most recent studies reported responder analysis $[50,53]$.

Risk of bias within studies. The methodological quality of the scientifically admissible studies is presented in Table 2. Most studies (5/6) had a low risk of bias with the exception of one which had a high or unclear risk of bias for the majority of the evaluated item (12/13) [52]. Among the low risk of bias studies, one publication provided an unclear description of the randomization [51] and another one did the same for the allocation concealment [43, 44]. As expected items related to the blinding of patients, care providers, and outcome assessors were at high risk of bias in every study due to the intervention and outcome considered in this review. Selective outcome reporting was suspected in one study reporting an unusual primary outcome [53]. Co-interventions use was different between groups in one study [45, 46] and unclear in another one $[43,44]$. Compliance was different between the two treatments group in one study [53] and could not be clearly evaluated in two studies [43, 44, 51]. The possibility of an additional bias was suspected in one study that restricted the number of treatment sessions in only one of its treatment groups $[43,44]$.

\section{Summary of evidence}

Chiropractic care vs exercise therapy care. Bronfort et al. [50] compared chiropractic care to exercise therapy in the United States for LBP subjects of at least six weeks duration. The extracted results are presented in Table 3. Outcomes of pain, functional status and global improvement assessed at one, three and 12 months revealed no significant difference between the two provider groups. The responder analysis (proportion of patients with at least $50 \%$ and $75 \%$ improvement) was coherent with the averaged results and no significant difference was 


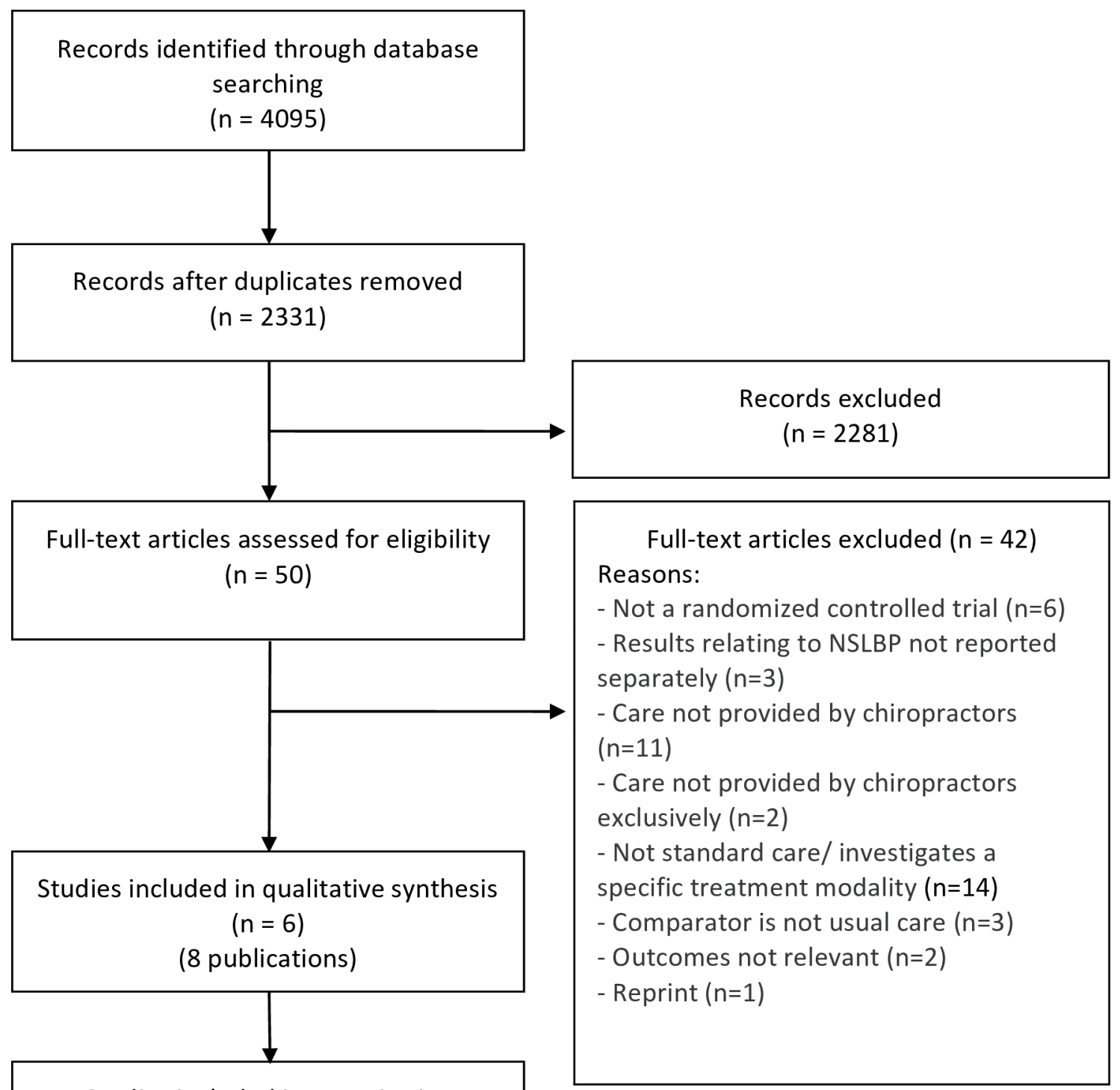

Studies included in quantitative synthesis (meta-analysis)

$(n=5)$

(7 publications)

Fig 1. Flow diagram for the selection of clinical effectiveness studies.

doi:10.1371/journal.pone.0160037.g001

found between the two types of provider for pain, functional status and global improvement assessed at three and 12 months.

Health related quality of life was assessed at one, three and 12 months using the physical and mental health subscales of the SF-36. The only statistically significant difference favour chiropractic care for the mental subscale at three months follow-up.

The rate of adverse effects did not differ significantly between the two treatment groups (Table 4$)$. These were rare (2\% and $6 \%$ for chiropractic and exercise therapy care respectively), transient in nature, and necessitated minimal or no change in activity level. 
Table 1. Characteristics of the clinical effectiveness studies included into the quantitative synthesis of Chiropractic care for non-specific low back pain.

\begin{tabular}{|c|c|c|c|c|}
\hline $\begin{array}{l}\text { First Author, Year, } \\
\text { Country and Setting }\end{array}$ & Participants and Indication & Comparative Treatments & Follow-up assessment & Relevant outcomes \\
\hline $\begin{array}{l}\text { - Bronfort 2011[50] } \\
\text { - USA, University } \\
\text { research clinic in } \\
\text { Bloomington, MN }\end{array}$ & $\begin{array}{l}18 \text { to } 65 \text { years old with } \\
\text { mechanical LBP of at least } \\
6 \text {-week duration with or } \\
\text { without radiating pain. (sub- } \\
\text { acute/chronic) }\end{array}$ & $\begin{array}{l}\text { - Chiropractic care once to twice } \\
\text { per week for } 15 \text { to } 30 \text { minutes } \\
\text { including: SMT and few minutes of } \\
\text { soft-tissue massage, ice, or heat } \\
\text { ( } n=100) \text {. } \\
\text { - Supervised exercise therapy } \\
\text { provided by exercise therapists } \\
(n=100) \text {. }\end{array}$ & $\begin{array}{l}- \text { Total: } 1 \text { year } \\
\text { - Relevant for this } \\
\text { review: } 1 \text { month (week } \\
\text { 4), } 3 \text { months (week 12), } \\
12 \text { months (week 52) }\end{array}$ & $\begin{array}{l}\text { - Functional status (Roland- } \\
\text { Morris 0-23) } \\
\text { - Health related quality of life } \\
\text { (SF-36 physical and mental } \\
\text { scales) } \\
\text { - Global improvement } \\
\text { (1= complete improvement, } \\
9=\text { twice as bad) } \\
\text { - Adverse events }\end{array}$ \\
\hline $\begin{array}{l}\text { - Cherkin } 1998 \text { [51] } \\
\text { - USA, Group Health } \\
\text { Cooperative of } \\
\text { Puget Sound (HMO), } \\
\text { Seattle, WA }\end{array}$ & $\begin{array}{l}\text { Patients } 20 \text { to } 64 \text { years of age } \\
\text { who saw their primary care } \\
\text { physician for low back pain } \\
\text { and who still had pain seven } \\
\text { days later. (mix/not specified) }\end{array}$ & $\begin{array}{l}\text { - Chiropractic care: according to } \\
\text { usual clinicians procedures } \\
\text { including recommendations about } \\
\text { exercise and activity restrictions } \\
\text { ( } n=122) \text {. } \\
\text { - Physical therapy care: provided by } \\
\text { therapists trained by the McKenzie } \\
\text { Institute faculty. Subjects received } \\
\text { McKenzie's Treat Your Own Back } \\
\text { book and a lumbar-support } \\
\text { cushion. Therapists were instructed } \\
\text { to avoid therapies such as heat, } \\
\text { ice, transcutaneous electrical nerve } \\
\text { stimulation, ultrasonography, and } \\
\text { back classes }(n=133) \text {. }\end{array}$ & $\begin{array}{l}\text { - Total: } 2 \text { years } \\
\text { - Relevant for this } \\
\text { review: } 1 \text { month (week } \\
\text { 4), } 3 \text { months } \\
\text { (week 12)* }\end{array}$ & $\begin{array}{l}\text { - Functional status (Roland- } \\
\text { Morris 0-24) } \\
\text { - Adverse events }\end{array}$ \\
\hline $\begin{array}{l}\text { - Herzog } 1991 \text { [52] } \\
\text { - Canada, Unknown } \\
\text { setting in Calgary, } \\
\text { Alberta }\end{array}$ & $\begin{array}{l}\text { Ambulatory patient between } \\
18 \text { and } 50 \text { years old with a } \\
\text { sacroiliac joint problem since } \\
\text { at least one month. (sub- } \\
\text { acute/chronic) }\end{array}$ & $\begin{array}{l}\text { - Chiropractic care: SMT and the } \\
\text { optimal treatment modality to the } \\
\text { discretion of the chiropractor** for } \\
10 \text { sessions over } 4 \text { week }(n=16) \text {. } \\
\text { - Physical therapy care: back school } \\
\text { therapy for } 10 \text { sessions over } 4 \\
\text { week }(n=13) \text {. }\end{array}$ & $\begin{array}{l}- \text { Total: } 1 \text { month (week 4, } \\
\text { treatment completion) } \\
\text { - Relevant for this } \\
\text { review: none }\end{array}$ & $\begin{array}{l}\text { - Actual pain (VAS 0-10) } \\
\text { - Functional status (Oswestry } \\
0-100 \text { ) }\end{array}$ \\
\hline $\begin{array}{l}\text { - Hurwitz } 2002[45,46] \\
\text { - USA, } 3 \text { Primary Care } \\
\text { Centers of a } 100000 \\
\text { member health-care } \\
\text { network based in } \\
\text { southern California }\end{array}$ & $\begin{array}{l}\text { HMO member of at least } 18 \\
\text { years old with a complaint of } \\
\text { low back pain with or without } \\
\text { leg pain. (mix/not specified) }\end{array}$ & $\begin{array}{l}\text { - Chiropractic Care: SMT, } \\
\text { instruction in strengthening and } \\
\text { flexibility exercises, and instruction } \\
\text { in proper back care }(n=169) \text {. } \\
\text { - Medical care: One or more of the } \\
\text { following: instruction in proper back } \\
\text { care and strengthening and } \\
\text { flexibility exercises; prescriptions } \\
\text { for pain killers, muscle relaxants, } \\
\text { anti-inflammatory agents, and other } \\
\text { medications used to reduce or } \\
\text { eliminate pain or discomfort; and } \\
\text { recommendations regarding bed } \\
\text { rest, weight loss, and physical } \\
\text { activities }(n=170) \text {. }\end{array}$ & $\begin{array}{l}- \text { Total: } 1.5 \text { year } \\
\text { - Relevant for this } \\
\text { review: } 1 \text { month (week } \\
\text { 6), } 12 \text { months }\end{array}$ & $\begin{array}{l}\text { - Average pain (VAS 0-10) } \\
\text { - Functional status (Roland- } \\
\text { Morris 0-24) } \\
\text { - Adverse events }\end{array}$ \\
\hline $\begin{array}{l}\text { - Meade } 1990[43,44] \\
\text { - United-Kingdom, } 11 \\
\text { centres with hospital } \\
\text { and chiropractic } \\
\text { clinics within a } \\
\text { reasonable distance }\end{array}$ & $\begin{array}{l}\text { Patients } 18 \text { to } 65 \text { years of age } \\
\text { with low back pain of } \\
\text { mechanical origin. (mix/not } \\
\text { specified) }\end{array}$ & $\begin{array}{l}\text { - Chiropractic care: at the discretion } \\
\text { of the chiropractor for a maximum } \\
\text { of } 10 \text { treatments over one year. The } \\
\text { treatments were intended to be } \\
\text { concentrated within the first } 3 \\
\text { months }(n=384) \text {. } \\
\text { - Physiotherapy care: within hospital } \\
\text { outpatient clinics }(n=357) \text {. }\end{array}$ & $\begin{array}{l}- \text { Total: } 3 \text { years } \\
\text { - Relevant for this } \\
\text { review: } 1 \text { month (week } \\
\text { 6), } 1 \text { year }\end{array}$ & $\begin{array}{l}\text { - Functional status (Oswestry } \\
0-100) \\
\text { - Global improvement (Number } \\
\text { of patients partially or } \\
\text { complete relieved) }\end{array}$ \\
\hline
\end{tabular}


Table 1. (Continued)

\begin{tabular}{|c|c|c|c|c|}
\hline $\begin{array}{l}\text { First Author, Year, } \\
\text { Country and Setting }\end{array}$ & Participants and Indication & Comparative Treatments & Follow-up assessment & Relevant outcomes \\
\hline $\begin{array}{l}\text { - Petersen 2011[53] } \\
\text { - Denmark, Primary } \\
\text { care specialist } \\
\text { center in } \\
\text { Copenhagen }\end{array}$ & $\begin{array}{l}\text { Patients of } 18 \text { and } 60 \text { years of } \\
\text { age suffering from LBP with } \\
\text { or without leg pain since more } \\
\text { than } 6 \text { weeks. (sub-acute/ } \\
\text { chronic) }\end{array}$ & $\begin{array}{l}\text { - Chiropractic care: all type of } \\
\text { manual } \\
\text { technique including SMT and } \\
\text { myofascial trigger-point massage at } \\
\text { the discretion of the chiropractor for } \\
\text { a maximum of } 15 \text { treatments in a } 12 \\
\text { weeks period. Mobilizing exercises, } \\
\text { alternating lumbar flexion/extension } \\
\text { movements, and stretching, were } \\
\text { allowed ( } n=175) \text {. } \\
\text { - Physical therapy care: according to } \\
\text { the McKenzie treatment protocols. } \\
\text { An educational booklet about self- } \\
\text { care or a "lumbar roll" for the seated } \\
\text { posture were sometimes provided } \\
\text { to the patient }(n=175) \text {. }\end{array}$ & $\begin{array}{l}\text { - Total: } 12 \text { months (post- } \\
\text { treatment completion) } \\
\text { - Relevant for this } \\
\text { review: } 3 \text { months } \\
\text { (treatment completion } \\
\text { (12 week)), } 12 \text { months } \\
\text { (post-treatment } \\
\text { completion) }\end{array}$ & $\begin{array}{l}\text { - Functional status (Roland- } \\
\text { Morris 0-23) } \\
\text { - Back and leg pain (0-60 scale } \\
\text { from } 6 \text { VAS (actual, worst, } \\
\text { average)) } \\
\text { - Health related quality of life } \\
\text { (SF-36 general health } \\
\text { perception and mental health } \\
\text { scales (0-100)) } \\
\text { - Global improvement (Number } \\
\text { of patients scoring completely } \\
\text { cured_or much improved on a } \\
\text { 6-point Likert scale (much } \\
\text { worse, worsened, no change, } \\
\text { improved, much improved, } \\
\text { completely cured)) }\end{array}$ \\
\hline
\end{tabular}

HMO: Health Maintenance Organization; SMT: Spinal Manipulative Therapy; VAS: Visual Analog Scale

* Results for the one-year follow-ups were only provided graphically and could not be used for this review

** Precisions regarding the chiropractic care modalities obtained from communication with the study authors

doi:10.1371/journal.pone.0160037.t001

Chiropractic care vs physical therapy care. Three studies compared chiropractic to physical therapy care; they were conducted in the United-States [51], United Kingdom [43, 44] and Denmark [53]. Two included a mix of LBP duration [43, 44, 51] and one included subjects with symptom onset of six weeks or more [53]. The extracted results and pooled effect are presented in Table 5 and Fig 2.

Pain was assessed at three and 12 months by only one study. There was no significant difference between the two treatment groups.

Functional status was assessed at 1 month $(n=2), 3$ months $(n=2)$ and 12 months $(n=2)$. None of the individual studies or the pooled effects showed significant between group differences. The only exception was the Danish study [53], in which a small statistically significant effect in favour of physical therapy care at the 12 months follow-up was reported. The responder analysis (number of patients with an absolute Roland Morris score below 5 points or at least 5 points reduction) of that study revealed no significant between group difference for

Table 2. Risk of bias of the included clinical effectiveness studies of chiropractic care for non-specific low back pain.

\begin{tabular}{|c|c|c|c|c|c|c|c|c|c|c|c|c|c|c|}
\hline & $\begin{array}{l}\text { Random } \\
\text { sequence } \\
\text { generation }\end{array}$ & $\begin{array}{l}\text { Allocation } \\
\text { concealment }\end{array}$ & $\begin{array}{l}\text { Blinding of } \\
\text { participants }\end{array}$ & $\begin{array}{l}\text { Blinding of } \\
\text { personnel } \\
\text { /care } \\
\text { providers }\end{array}$ & $\begin{array}{l}\text { Blinding of } \\
\text { outcomes } \\
\text { assessors }\end{array}$ & $\begin{array}{l}\text { Incomplete } \\
\text { outcome } \\
\text { data }\end{array}$ & $\begin{array}{l}\text { Selective } \\
\text { outcome } \\
\text { reporting }\end{array}$ & $\begin{array}{l}\text { Group } \\
\text { similarity } \\
\text { at } \\
\text { baseline }\end{array}$ & $\begin{array}{l}\text { Co- } \\
\text { interventions }\end{array}$ & Compliance & $\begin{array}{l}\text { Intention- } \\
\text { to-treat- } \\
\text { analysis }\end{array}$ & $\begin{array}{l}\text { Timing of } \\
\text { outcome } \\
\text { assessments }\end{array}$ & $\begin{array}{l}\text { Other } \\
\text { bias }\end{array}$ & $\begin{array}{l}\text { Overall } \\
\text { risk of } \\
\text { bias }\end{array}$ \\
\hline $\begin{array}{l}\text { Bronfort } \\
2011[50]\end{array}$ & Low & Low & High & High & High & Low & Low & Low & Low & Low & Low & Low & Low & Low \\
\hline $\begin{array}{l}\text { Cherkin } \\
1998 \text { [51] }\end{array}$ & Unclear & Low & High & High & High & Low & Low & Low & Low & Unclear & Low & Low & Low & Low \\
\hline $\begin{array}{l}\text { Herzog } \\
1991 \text { [52] }\end{array}$ & Unclear & Unclear & High & High & High & High & Unclear & High & Low & Unclear & High & High & High & High \\
\hline $\begin{array}{l}\text { Hurwitz } \\
2002[45, \\
46]\end{array}$ & Low & Low & High & High & High & Low & Low & Low & High & Low & Low & Low & Low & Low \\
\hline $\begin{array}{l}\text { Meade } \\
1990[43, \\
44]\end{array}$ & Low & Unclear & High & High & High & Low & Low & Low & Unclear & Unclear & Low & Low & High & Low \\
\hline $\begin{array}{l}\text { Petersen } \\
2011[53]\end{array}$ & Low & Low & High & High & High & Low & High & Low & Low & High & Low & Low & Low & Low \\
\hline
\end{tabular}

doi:10.1371/journal.pone.0160037.t002 
Table 3. Chiropractic care versus Exercise therapist care.

\begin{tabular}{|c|c|c|c|c|c|c|c|c|c|c|c|c|}
\hline \multirow[b]{2}{*}{ Study } & \multirow[b]{2}{*}{ Outcome } & \multirow[b]{2}{*}{ Time } & \multicolumn{4}{|c|}{ Chiropractic } & \multicolumn{4}{|c|}{ Exercise therapist } & \multicolumn{2}{|l|}{ Overall } \\
\hline & & & Mean & SD & $\mathbf{N}$ & $\begin{array}{l}\% \text { of change } \\
\text { from baseline }\end{array}$ & Mean & SD & $\mathbf{N}$ & $\begin{array}{l}\% \text { of change } \\
\text { from baseline }\end{array}$ & $\begin{array}{l}\text { Standardized mean } \\
\text { difference }(95 \% \mathrm{Cl})\end{array}$ & $\begin{array}{c}P- \\
\text { value }\end{array}$ \\
\hline \multirow{15}{*}{$\begin{array}{l}\text { Bronfort } 2011 \\
\text { (sub-acute/ } \\
\text { chronic) }\end{array}$} & \multirow[t]{3}{*}{ Pain } & $\begin{array}{l}1 \\
\text { month }\end{array}$ & 3.9 & 1.8 & 100 & -27.8 & 3.7 & 1.8 & 95 & -27.5 & $0.11(-0.17,0.39)$ & 0.44 \\
\hline & & $\begin{array}{l}3 \\
\text { month }\end{array}$ & 2.9 & 1.9 & 99 & -46.3 & 2.6 & 2.1 & 93 & -49.0 & $0.15(-0.13,0.43)$ & 0.30 \\
\hline & & $\begin{array}{l}12 \\
\text { month }\end{array}$ & 3.3 & 2.1 & 81 & -38.9 & 2.8 & 2.3 & 82 & -45.1 & $0.23(-0.08,0.53)$ & 0.15 \\
\hline & \multirow[t]{3}{*}{ Functional status } & $\begin{array}{l}1 \\
\text { month }\end{array}$ & 5.9 & 4.9 & 100 & -32.2 & 5.9 & 4.4 & 94 & -29.8 & $0.00(-0.28,0.28)$ & 1.00 \\
\hline & & $\begin{array}{l}3 \\
\text { month }\end{array}$ & 4.9 & 5.0 & 99 & -43.7 & 3.9 & 4.6 & 92 & -53.6 & $0.21(-0.08,0.49)$ & 0.15 \\
\hline & & $\begin{array}{l}12 \\
\text { month }\end{array}$ & 5.1 & 4.9 & 81 & -41.4 & 3.8 & 4.7 & 82 & -54.8 & $0.27(-0.04,0.58)$ & 0.09 \\
\hline & \multirow{3}{*}{$\begin{array}{l}\text { Health related quality of } \\
\text { life (SF-36 Physical } \\
\text { scale) }\end{array}$} & $\begin{array}{l}1 \\
\text { month }\end{array}$ & 46.2 & 7.1 & 100 & 7.9 & 47.2 & 8.0 & 94 & 8.0 & $-0.13(-0.41,0.15)$ & 0.36 \\
\hline & & $\begin{array}{l}3 \\
\text { month }\end{array}$ & 48.0 & 7.7 & 99 & 12.1 & 49.7 & 7.8 & 92 & 13.7 & $-0.22(-0.50,0.07)$ & 0.13 \\
\hline & & $\begin{array}{l}12 \\
\text { month }\end{array}$ & 48.4 & 8.0 & 81 & 13.1 & 50.4 & 7.2 & 82 & 15.3 & $-0.26(-0.57,0.05)$ & 0.10 \\
\hline & \multirow[t]{3}{*}{$\begin{array}{l}\text { Health related quality of } \\
\text { life (SF-36 Mental scale) }\end{array}$} & $\begin{array}{l}1 \\
\text { month }\end{array}$ & 56.0 & 6.7 & 100 & 1.6 & 53.9 & 9.1 & 94 & 0.4 & $0.26(-0.02,0.55)$ & 0.07 \\
\hline & & $\begin{array}{l}3 \\
\text { month }\end{array}$ & 57.2 & 5.3 & 99 & 3.8 & 55.2 & 7.8 & 92 & 2.8 & $0.30(0.02,0.59)$ & 0.04 \\
\hline & & $\begin{array}{l}12 \\
\text { month }\end{array}$ & 55.2 & 7.5 & 81 & 0.2 & 53.9 & 8.6 & 82 & 0.4 & $0.16(-0.15,0.47)$ & 0.31 \\
\hline & \multirow[t]{3}{*}{ Global improuvement } & $\begin{array}{l}1 \\
\text { month }\end{array}$ & 3.5 & 1.2 & 100 & Not available & 3.8 & 1.0 & 94 & Not available & $-0.27(-0.55,0.01)$ & 0.06 \\
\hline & & $\begin{array}{l}3 \\
\text { month }\end{array}$ & 2.9 & 1.4 & 99 & Not available & 2.7 & 1.3 & 92 & Not available & $0.15(-0.14,0.43)$ & 0.31 \\
\hline & & $\begin{array}{l}12 \\
\text { month }\end{array}$ & 3.3 & 1.6 & 81 & Not available & 3.1 & 1.6 & 82 & Not available & $0.12(-0.18,0.43)$ & 0.43 \\
\hline
\end{tabular}

doi:10.1371/journal.pone.0160037.t003

functional status at three and 12 months. This study was the only one to report functional status at 12 months for subacute/chronic subpopulation. Nevertheless, the pooled effect, including results of the Meade study [43,44] from a mixed subpopulation, did not reveal significant between group differences. The heterogeneity analysis of the pooled three and 12 months outcomes showed substantial heterogeneity. Potential sources were: different settings (USA, UK and Denmark), different subpopulations in terms of symptom duration, and different physical therapy approaches (McKenzie method) and usual hospital outpatient clinic.

Global improvement was assessed at one and three months in one study. At one month, Meade et al. [43, 44] reported a statistically significant advantage in favour of chiropractic care, while at three months Petersen et al. [53] reported a statistically significant advantage in favour of physical therapy care.

Health related quality of life was only reported by Petersen et al. [53] at three and 12 months using the general health perception and mental health subscales of the SF-36. None of the comparisons significantly statistically favoured one type of care.

Return to work was also reported in the Danish study [53]. Since the two treatment groups were significantly different regarding their number of participants off work at baseline, we chose not to extract data for that particular outcome. 
Table 4. Adverse events.

\begin{tabular}{|c|c|c|c|c|c|c|}
\hline Study & $\begin{array}{l}\text { Adverse } \\
\text { events }\end{array}$ & $\mathbf{N}$ & $\begin{array}{l}\text { Adverse } \\
\text { events }\end{array}$ & $\mathbf{N}$ & $\begin{array}{c}\text { Risk Ratio }(95 \% \\
\text { Cl) }\end{array}$ & P-value \\
\hline \multirow{2}{*}{$\begin{array}{l}\text { Bronfort } 2011 \text { (sub-acute/ } \\
\text { chronic) }\end{array}$} & \multicolumn{2}{|c|}{ Chiropractic } & \multicolumn{2}{|c|}{ Exercise therapist } & & \\
\hline & 2 & 100 & 6 & 100 & $0.33(0.07,1.61)$ & 0.17 \\
\hline \multirow{2}{*}{$\begin{array}{l}\text { Cherkin } 1998 \text { (mix/not } \\
\text { specified) }\end{array}$} & \multicolumn{2}{|c|}{ Chiropractic } & \multicolumn{2}{|c|}{ Physical therapy } & & \\
\hline & 0 & 122 & 0 & 133 & Not estimable & $\begin{array}{c}\text { Not } \\
\text { applicable }\end{array}$ \\
\hline \multirow{2}{*}{$\begin{array}{l}\text { Hurwitz } 2002 \text { (mix/not } \\
\text { specified) }\end{array}$} & \multicolumn{2}{|c|}{ Chiropractic } & \multicolumn{2}{|c|}{ Medical } & & \\
\hline & 0 & 169 & 0 & 170 & Not estimable & $\begin{array}{c}\text { Not } \\
\text { applicable }\end{array}$ \\
\hline
\end{tabular}

doi:10.1371/journal.pone.0160037.t004

Adverse events were only reported in the Cherkin study [51], and no serious adverse effects were recorded in either of the treatment groups (Table 4).

Chiropractic care vs medical care. Only one study compared chiropractic care to medical care. The study was conducted by Hurwitz et al. in the United States and included subjects with a mixed symptom duration of LBP $[45,46]$. Extracted results for this study are presented into Table 6.

Pain and functional status were assessed at one and 12 months. There was no significant difference between the two treatment groups.

Global improvement, health related quality of life, and return to work were not assessed. No serious adverse events were recorded in neither of the treatment group (Table 4).

Overall, the pooled results did not show significant differences in effectiveness between chiropractic care and the other type of standard care studied. When reported, no serious adverse events were recorded in any of the chiropractic, exercise therapy [50], physical therapy [51] or medical $[45,46]$ treatment groups (Table 4).

\section{Economic evaluation}

Study selection. Our search yielded 2,725 citations. We removed 905 duplicates and screened 1,820 articles for eligibility (Fig 3). After screening, 1,761 articles did not meet our selection criteria. Fifty-nine articles were assessed for eligibility and only three articles were scientifically admissible. The main reasons for exclusion of the full papers were: partial economic evaluations or lack of any cost evaluation.

Study characteristics. The characteristics of these included studies are presented in Table 7. All three included studies were conducted in the United-States, included between 417 and 2780 participants, and involved follow-up periods ranging from 1 to 3.7 years. The types of economic evaluation performed were: cost-effectiveness analysis $(n=1)$ [54], cost-benefits analysis $(n=1)$ [55], and cost-minimizations analysis conducted along with a randomized clinical trial $(n=1)$ [56]. All three included studies compared chiropractic care to combinations of medical care. The quality assessment of the included studies is reported in Table 8.

Summary of evidence and quality assessment. Butler et al. [55] conducted a cost-benefit study in the US from the perspective of the employer as a proxy of the societal perspective. The authors used data from a prospective cohort study as an exemplar to present a method for adjusting rehabilitation costs and benefits for health capital. The cohort consisted of patients with occupational low back pain from five employers across 37 federal states. Chiropractic care $(\mathrm{n}=15$ patients) was compared to different types of providers: medical doctors $(\mathrm{n}=20$ patients), medical doctors and physical therapists (MDPt, $\mathrm{n}=144$ patients), chiropractors and medical doctors ( $\mathrm{n}=105$ patients), and surgeons or emergency physicians ( $\mathrm{n}=133$ patients). 
Table 5. Chiropractic care versus Physical therapy care.

\begin{tabular}{|c|c|c|c|c|c|c|c|c|c|c|c|c|}
\hline \multirow[b]{2}{*}{ Outcome } & \multirow[b]{2}{*}{ Time } & \multirow[b]{2}{*}{ Study } & \multicolumn{4}{|c|}{ Chiropractic } & \multicolumn{4}{|c|}{ Physical therapy } & \multicolumn{2}{|l|}{ Overall } \\
\hline & & & Mean & SD & $\mathbf{N}$ & $\begin{array}{l}\% \text { of change } \\
\text { from baseline }\end{array}$ & Mean & SD & $\mathbf{N}$ & $\begin{array}{c}\% \text { of change } \\
\text { from baseline }\end{array}$ & $\begin{array}{l}\text { Standardized mean } \\
\text { difference }(95 \% \mathrm{Cl})\end{array}$ & $\begin{array}{c}\mathrm{P}- \\
\text { value }\end{array}$ \\
\hline \multirow[t]{2}{*}{$\begin{array}{l}\text { Pain reduction from } \\
\text { baseline }\end{array}$} & $\begin{array}{l}3 \\
\text { month }\end{array}$ & $\begin{array}{l}\text { Petersen } 2011 \\
\text { (sub-acute/ } \\
\text { chronic) }\end{array}$ & 13.8 & 13.0 & 163 & -47.4 & 15.4 & 13.4 & 172 & -51.2 & $-0.12(-0.33,0.09)$ & 0.27 \\
\hline & $\begin{array}{l}12 \\
\text { month }\end{array}$ & $\begin{array}{l}\text { Petersen } 2011 \\
\text { (sub-acute/ } \\
\text { chronic) }\end{array}$ & 12.2 & 13.7 & 163 & -42.1 & 15.0 & 13.6 & 161 & -50.0 & $-0.20(-0.42,0.01)$ & 0.07 \\
\hline \multirow[t]{6}{*}{ Functional status } & \multirow[t]{2}{*}{$\begin{array}{l}1 \\
\text { month }\end{array}$} & $\begin{array}{l}\text { Meade } 1990 \\
\text { (mix/not } \\
\text { specified) }\end{array}$ & 15.8 & 16.0 & 357 & -47.0 & 17.5 & 16.0 & 309 & -38.6 & \multirow[t]{2}{*}{$-0.10(-0.23,0.03)$} & \multirow[t]{2}{*}{0.13} \\
\hline & & $\begin{array}{l}\text { Cherkin } 1998 \\
\text { (mix/not } \\
\text { specified) }\end{array}$ & 3.7 & 4.4 & 118 & -69.4 & 4.1 & 4.6 & 129 & -66.4 & & \\
\hline & \multirow[t]{2}{*}{$\begin{array}{l}3 \\
\text { month }\end{array}$} & $\begin{array}{l}\text { Cherkin } 1998 \\
\text { (mix/not } \\
\text { specified) }\end{array}$ & 3.1 & 4.2 & 118 & -74.4 & 4.1 & 5.0 & 117 & -66.4 & \multirow[t]{2}{*}{$-0.04(-0.37,0.28)$} & \multirow[t]{2}{*}{0.80} \\
\hline & & $\begin{array}{l}\text { Petersen } 2011 \\
\text { (sub-acute/ } \\
\text { chronic) }\end{array}$ & 7.2 & 6.1 & 163 & -44.6 & 6.5 & 6.1 & 172 & -50.0 & & \\
\hline & \multirow[t]{2}{*}{$\begin{array}{l}12 \\
\text { month }\end{array}$} & $\begin{array}{l}\text { Petersen } 2011 \\
\text { (sub-acute/ } \\
\text { chronic) }\end{array}$ & 7.4 & 6.0 & 163 & -43.1 & 5.9 & 6.0 & 161 & -54.6 & \multirow[t]{2}{*}{$0.06(-0.31,0.42)$} & \multirow[t]{2}{*}{0.76} \\
\hline & & $\begin{array}{l}\text { Meade } 1990 \\
\text { (mix/not } \\
\text { specified) }\end{array}$ & 15.3 & 16.8 & 314 & -48.7 & 17.3 & 16.8 & 265 & -39.2 & & \\
\hline \multirow[t]{2}{*}{$\begin{array}{l}\text { Health related } \\
\text { quality of life } \\
\text { (General) }\end{array}$} & $\begin{array}{l}3 \\
\text { month }\end{array}$ & $\begin{array}{l}\text { Petersen } 2011 \\
\text { (sub-acute/ } \\
\text { chronic) }\end{array}$ & 69.5 & 19.6 & 163 & 6.9 & 72.1 & 19.6 & 172 & 7.6 & $-0.13(-0.35,0.08)$ & 0.23 \\
\hline & $\begin{array}{l}12 \\
\text { month }\end{array}$ & $\begin{array}{l}\text { Petersen } 2011 \\
\text { (sub-acute/ } \\
\text { chronic) }\end{array}$ & 65.3 & 23.0 & 163 & 0.5 & 69.5 & 23.0 & 161 & 3.7 & $-0.18(-0.40,0.04)$ & 0.10 \\
\hline \multirow[t]{3}{*}{$\begin{array}{l}\text { Health related } \\
\text { quality of life } \\
\text { (Mental) }\end{array}$} & $\begin{array}{l}3 \\
\text { month }\end{array}$ & $\begin{array}{l}\text { Petersen } 2011 \\
\text { (sub-acute/ } \\
\text { chronic) }\end{array}$ & 74.2 & 20.2 & 163 & 14.2 & 74.2 & 20.2 & 172 & 14.2 & $0.00(-0.21,0.21)$ & 0.99 \\
\hline & $\begin{array}{l}12 \\
\text { month }\end{array}$ & $\begin{array}{l}\text { Petersen } 2011 \\
\text { (sub-acute/ } \\
\text { chronic) }\end{array}$ & 73.8 & 20.4 & 163 & 13.5 & 76.2 & 20.4 & 161 & 17.2 & $-0.12(-0.34,0.10)$ & 0.29 \\
\hline & & & \multicolumn{2}{|c|}{ Events } & \multicolumn{2}{|r|}{$\mathbf{N}$} & \multicolumn{2}{|c|}{ Events } & \multicolumn{2}{|r|}{$\mathbf{N}$} & Risk Ratio $(95 \% \mathrm{Cl})$ & $\begin{array}{c}\mathrm{P}- \\
\text { value }\end{array}$ \\
\hline \multirow[t]{2}{*}{$\begin{array}{l}\text { Global } \\
\text { improuvement }\end{array}$} & $\begin{array}{l}1 \\
\text { month }\end{array}$ & $\begin{array}{l}\text { Meade } 1990 \\
\text { (mix/not } \\
\text { specified) }\end{array}$ & \multicolumn{2}{|c|}{312} & & 360 & \multicolumn{2}{|c|}{245} & & 317 & $1.12(1.04,1.21)$ & 0.002 \\
\hline & $\begin{array}{l}3 \\
\text { month }\end{array}$ & $\begin{array}{l}\text { Petersen } 2011 \\
\text { (sub-acute/ } \\
\text { chronic) }\end{array}$ & \multicolumn{2}{|c|}{53} & & 153 & \multicolumn{2}{|c|}{81} & & 169 & $0.72(0.55,0.95)$ & 0.02 \\
\hline
\end{tabular}

doi:10.1371/journal.pone.0160037.t005

Direct costs (offices visits, consultations, physical medicine, radiographs, medication) and indirect costs (saving from work loss day) were considered. Costs related to the household sector were not included. Based on the nearly identical adjusted net benefits for physician only care $(135,824 \$)$, physician plus physical therapy care $(130,064 \$)$, and chiropractic care $(132,989 \$)$, the three types of care seemed equivalent. Net benefits of care were lower for combined physician/chiropractic care $(104,025 \$)$, and lowest for all other forms of care $(104,025 \$)$. Precision of the estimates and sensitivity analysis were not reported. Butler et al. presented the analysis as 


\section{Functional Status at 1 month}

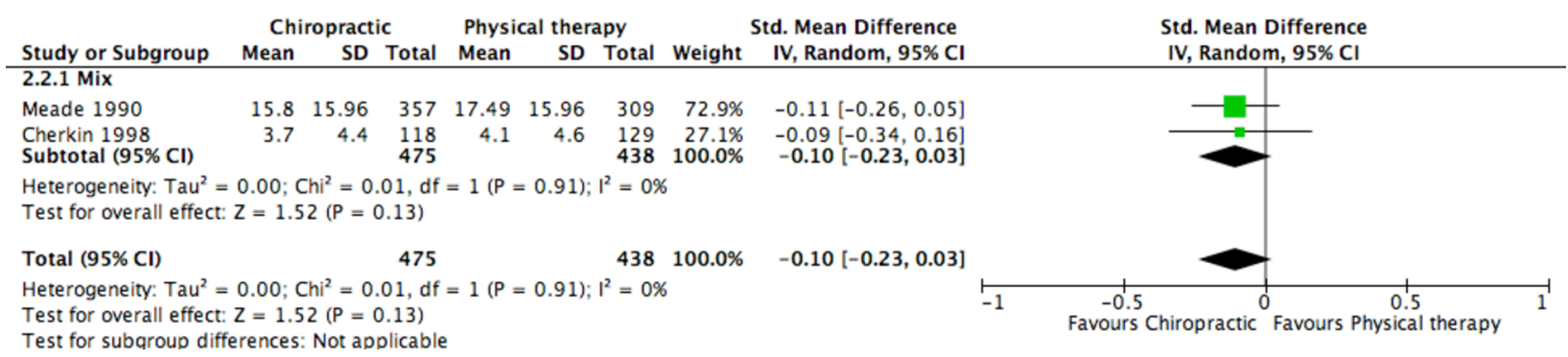

\section{Functional Status at 3 months}

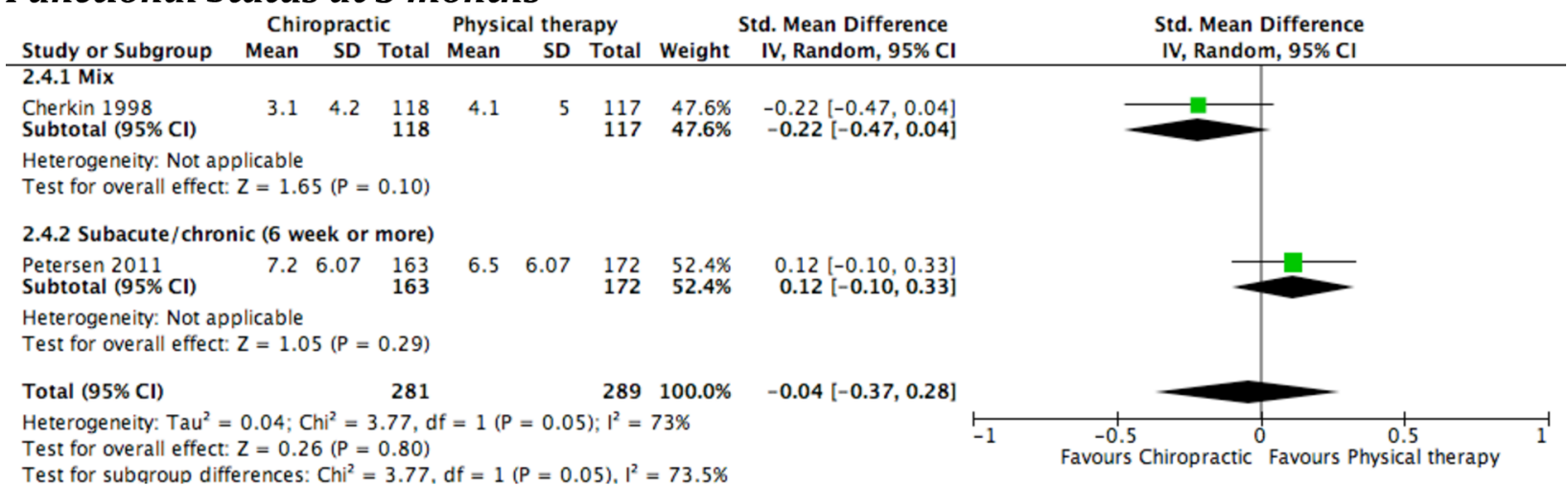

\section{Functional Status at 12 months}

\begin{tabular}{|c|c|c|c|c|c|c|c|c|c|c|c|}
\hline \multirow[b]{2}{*}{ Study or Subgroup } & \multicolumn{3}{|c|}{ Chiropractic } & \multicolumn{3}{|c|}{ Physical therapy } & \multicolumn{2}{|r|}{ Std. Mean Difference } & \multirow{2}{*}{\multicolumn{3}{|c|}{$\begin{array}{l}\text { Std. Mean Difference } \\
\text { IV, Random, } 95 \% \mathrm{Cl}\end{array}$}} \\
\hline & Mean & SD & Total & Mean & SD & Total & Weight & IV, Random, 95\% Cl & & & \\
\hline \multicolumn{12}{|c|}{ 2.9.1 Subacute/chronic (6 week or more) } \\
\hline $\begin{array}{l}\text { Petersen } 2011 \\
\text { Subtotal }(95 \% \mathrm{CI})\end{array}$ & 7.4 & 5.97 & $\begin{array}{l}163 \\
163\end{array}$ & 5.9 & 5.97 & $\begin{array}{l}161 \\
161\end{array}$ & $\begin{array}{l}48.0 \% \\
48.0 \%\end{array}$ & $\begin{array}{l}0.25[0.03,0.47] \\
0.25[0.03,0.47]\end{array}$ & & & \\
\hline \multicolumn{12}{|c|}{$\begin{array}{l}\text { Heterogeneity: Not applicable } \\
\text { Test for overall effect: } Z=2.25(P=0.02)\end{array}$} \\
\hline \multicolumn{12}{|l|}{ 2.9.2 Mix } \\
\hline $\begin{array}{l}\text { Meade } 1990 \\
\text { Subtotal }(95 \% \mathrm{Cl})\end{array}$ & 15.3 & 16.82 & $\begin{array}{l}314 \\
314\end{array}$ & 17.34 & 16.82 & $\begin{array}{l}265 \\
265\end{array}$ & $\begin{array}{l}52.0 \% \\
52.0 \%\end{array}$ & $\begin{array}{l}-0.12[-0.28,0.04] \\
-0.12[-0.28,0.04]\end{array}$ & & & \\
\hline \multicolumn{12}{|c|}{$\begin{array}{l}\text { Heterogeneity: Not applicable } \\
\text { Test for overall effect: } Z=1.45(P=0.15)\end{array}$} \\
\hline Total $(95 \% \mathrm{Cl})$ & & & 477 & & & 426 & $100.0 \%$ & $0.06[-0.31,0.42]$ & & & \\
\hline \multicolumn{9}{|c|}{$\begin{array}{l}\text { Heterogeneity: } \mathrm{Tau}^{2}=0.06 ; \mathrm{Ch}^{2}=7.12, \mathrm{df}=1(\mathrm{P}=0.008) ; \mathrm{I}^{2}=86 \% \\
\text { Test for overall effect: } \mathrm{Z}=0.31(\mathrm{P}=0.76) \\
\text { Test for subarous differences: } \mathrm{Chi}^{2}=7.12 . \mathrm{df}=1(\mathrm{P}=0.008), \mathrm{I}^{2}=86.0 \%\end{array}$} & $\begin{array}{c}-1 \\
-0.5 \\
\text { Favours Chir }\end{array}$ & (n) & 0.5 \\
\hline
\end{tabular}

Fig 2. Forest plots of comparison: Chiropractic care versus Physical therapy care.

doi:10.1371/journal.pone.0160037.g002

an exemplar and therefore the sample size in the chiropractic group was low. With only 15 patients receiving chiropractic care, the parametric multivariate analyses conducted by the author is likely underpowered and their conclusions therefore not robust. Based on this, we rated the study quality as low despite the high quality of their methodology.

Haas et al. [54] conducted a cost effectiveness study from the perspective of the healthcare system (Medicare) based on a prospective cohort study. They recruited participants from 51 chiropractic clinics ( $\mathrm{n}=60$ providers and $\mathrm{n}=1855$ patients) and 14 general practice community clinics ( $\mathrm{n}=111$ medical providers and $\mathrm{n}=925$ patients $)$ in Oregon and Washington, US. The effectiveness measures were based on patient- reported outcomes including pain, functional status, treatment satisfaction, and physical and mental health. The following direct costs 
Table 6. Chiropractic care versus Medical care.

\begin{tabular}{c|l|l|r|r|r|r|r|r|r|r|}
\hline & & \multicolumn{3}{|c|}{ Chiropractic } & \multicolumn{3}{c|}{ Medical } & \multicolumn{2}{c}{ Overall } \\
\hline \multicolumn{1}{c|}{ Study } & Outcome & Time & Mean & SD & N & Mean & SD & N & $\begin{array}{l}\text { Standardized mean } \\
\text { difference (95\% CI) }\end{array}$ & $\begin{array}{c}\text { P- } \\
\text { value }\end{array}$ \\
\hline $\begin{array}{l}\text { Hurwitz 2002 } \\
\text { (mix/not } \\
\text { specified) }\end{array}$ & Pain & $\begin{array}{l}1 \\
\text { month }\end{array}$ & 3.6 & 2.58 & 169 & 3.86 & 2.58 & 169 & $-0.10(-0.31,0.11)$ & 0.36 \\
\cline { 2 - 11 } & $\begin{array}{l}12 \\
\text { month }\end{array}$ & 3.1 & 2.54 & 153 & 3.31 & 2.54 & 153 & $-0.08(-0.31,0.14)$ & 0.47 \\
\cline { 2 - 11 } & $\begin{array}{l}\text { Functional } \\
\text { status }\end{array}$ & $\begin{array}{l}1 \\
\text { month }\end{array}$ & 7.5 & 5.91 & 169 & 7.87 & 5.91 & 169 & $-0.06(-0.28,0.15)$ & 0.57 \\
\cline { 2 - 10 } & $\begin{array}{l}12 \\
\text { month }\end{array}$ & 7.05 & 6.02 & 153 & 7.00 & 6.02 & 153 & $0.01(-0.22,0.23)$ & 0.94 \\
\hline
\end{tabular}

doi:10.1371/journal.pone.0160037.t006

were considered: office visits, radiographs and medication. The costs related to advance imaging [57], surgical [58] and physical therapy consultations [51] were imputed from previous published studies. The services provided were assigned Current Procedural Terminology (CPT) codes that were converted to Medicare relative value units, and a conversion factor was applied to estimate the Medicare payment. The adjusted mean differences (AMDs) of total cost at 12 months revealed that chiropractic care was slightly more expensive than medical care for acute $(63 \$, P=0.352)$ and chronic patients $(1.5 \$, p=0.993)$, but the differences were not significant. The AMDs for pain and functional status at 12 months significantly advantaged chiropractic care, but were not clinically important (AMDs $<10, \mathrm{P}<0.01$ ). The ICER ranged from $4.5 \$$ to $23.6 \$$ per unit of change of the considered outcomes for the acute patients and from 0.0 $\$$ to $4.5 \$$ for the chronic patients. Formal willingness to pay and sensitivity analysis were not reported. Given the very small ICER for the chronic patients, chiropractic care appears relatively cost effective. The interpretation is less clear for acute patients, but chiropractic and medical care appear to perform comparably. Our overall assessment of the study lead us to rate this study as high quality, because it considered all the costs and outcomes relevant to the perspective of the analysis.

Kominski et al. [56] conducted a cost-minimization analysis alongside the randomized controlled trial reported by Hurwitz[45, 46] from the perspective of the healthcare provider group. The authors recruited Health Maintenance Organization (HMO) patients from three large medical group practices in Southern California, US. Chiropractic care $(n=162)$ was compared to medical care $(n=162)$, medical care combined with physical therapy $(n=167)$ and chiropractic care combined with physical modalities $(n=163)$. The medical group received capitated payments from the insurer. The costs in this study were imputed using CPT codes and a Medicare fee schedule. The following direct costs were considered: offices visits, diagnostic and therapeutic services. The mean total cost for chiropractic care $(769 \$)$ was significantly higher than medical care $(647 \$)$ without producing significantly better clinical outcomes. The addition of physical therapy $(1070 \$)$ to medical care and physical modalities to chiropractic care $(790 \$)$ also generated greater costs for similar clinical outcomes. Sensitivity analyses were not reported. The study quality was rated as medium, because the healthcare provider group used a remuneration system that likely differed from the Medicare one. As a consequence, the method used for cost imputation might not reflect the actual cost of the healthcare provider group. Additionally, medication costs were not considered. Inclusion of these costs might considerably influence the overall care cost, and in particular the medical care costs, and thereby the study conclusions.

In summary, according to the Slavin's best-evidence synthesis approach, the level of evidence of the economic findings relating to chiropractic compared to medical care is mixed as the three included studies reported inconsistent conclusions regarding chiropractic care. 


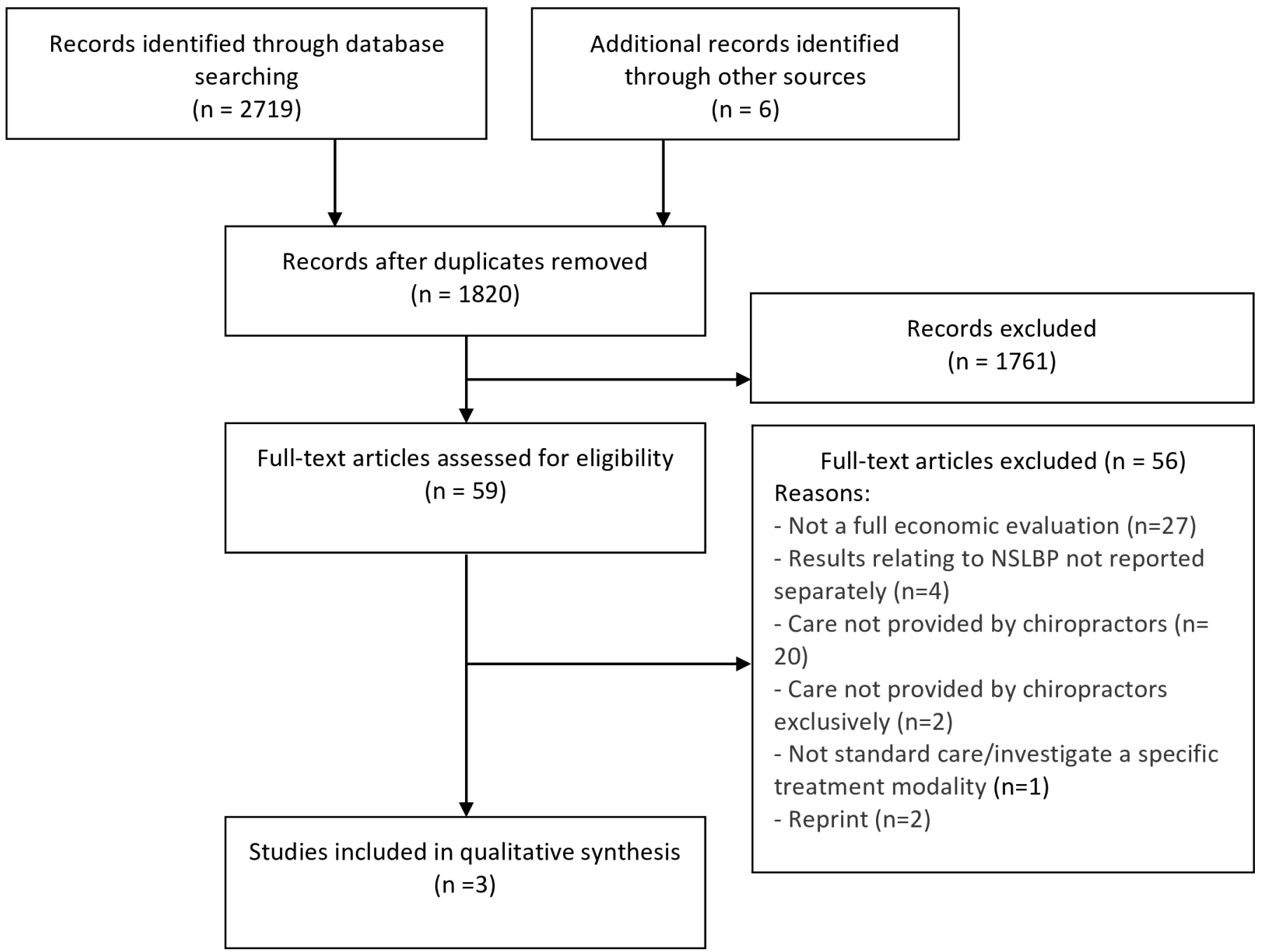

Fig 3. Flow diagram for the selection of economic evaluations.

doi:10.1371/journal.pone.0160037.g003

\section{Discussion}

\section{Summary of findings}

We identified six clinical effectiveness RCTs and three full economic evaluations. Overall, individual studies showed similar effects of chiropractic care compared to exercise therapy, physical therapy or medical care for the treatment of low back pain regardless of type of outcome, and the risk of bias was low in five out of six included studies. Similarly, the pooled results revealed no significant difference in effectiveness between providers groups. No serious adverse events were reported for any treatment approaches. The three full economic evaluation studies comparing chiropractic care to medical care were of low to high methodological quality. These studies provided conflicting results regarding cost-effectiveness, and the resulting level of evidence is mixed.

Unfortunately, very few studies met our inclusion criteria and all our effect estimates regarding primary and secondary outcomes came from one or two studies. Additionally, two of our pooled estimates included a considerable level of heterogeneity [59]. Therefore, future high quality studies are likely to influence effect estimates. In three of the included clinical effectiveness studies, we found individual outcomes that were not in concordance with our overall 
Table 7. Characteristics, key findings of economic evaluations of Chiropractic care for non-specific low back pain.

\begin{tabular}{|c|c|c|c|c|c|c|}
\hline $\begin{array}{l}\text { First Author, Year, } \\
\text { Country, Type of } \\
\text { economic evaluation }\end{array}$ & $\begin{array}{l}\text { Participants, Indication } \\
\text { and Setting }\end{array}$ & $\begin{array}{l}\text { Comparative } \\
\text { Treatments }\end{array}$ & $\begin{array}{l}\text { Perspective, Time } \\
\text { Horizon, Currency } \\
\text { Price (Year) }\end{array}$ & $\begin{array}{l}\text { Included Costs, } \\
\text { Health Effects }\end{array}$ & $\begin{array}{l}\text { Mean Health } \\
\text { effect, Mean Costs } \\
(2015 \text { USD })^{*}\end{array}$ & $\begin{array}{l}\text { Incremental Cost- } \\
\text { effectiveness, } \\
\text { Incremental net- } \\
\text { benefit }\end{array}$ \\
\hline \multirow{4}{*}{$\begin{array}{l}\text { - Butler, } 2010 \text { [55] } \\
\text { - US } \\
\text { - Cost-benefit } \\
\text { analysis/prospective } \\
\text { cohort }\end{array}$} & \multirow{4}{*}{$\begin{array}{l}\text { - } 417 \text { adults workers } \\
\text { - Occupational LBP } \\
\text { - Five employers with } \\
\text { establishments in } 37 \\
\text { states recruited for The } \\
\text { Arizona State University } \\
\text { Healthy Back Study }\end{array}$} & \multirow{4}{*}{$\begin{array}{l}\text { - Medical doctors or } \\
\text { osteopaths (MD/DO) } \\
(n=20) \\
\text { - MD/DO combined } \\
\text { with Physical therapy } \\
\text { (MDPt)( } n=144) \\
\text { - Chiropractors (DC) } \\
(n=15) \\
\text { - DC and MD/DOs } \\
(n=105) \\
\text { - Surgeons and MDs } \\
\text { in emergency } \\
\text { departments (Sx/ED) } \\
\text { ( } n=133)\end{array}$} & \multirow{4}{*}{$\begin{array}{l}\text { - Employer (society) } \\
\text { - } 3.7 \text { years } \\
\text { - } 2002 \text { US dollars }\end{array}$} & Costs: & $\begin{array}{l}\text { Adjusted Health } \\
\text { effects: }\end{array}$ & $\begin{array}{l}\text { Adjusted Net- } \\
\text { benefits: }\end{array}$ \\
\hline & & & & $\begin{array}{l}\text { - Offices visits } \\
\text { - Consultations } \\
\text { - Physical medicine } \\
\text { - X-rays } \\
\text { - Medication }\end{array}$ & $\begin{array}{l}\text { - MD/DO: } \\
\text { 147,113\$ } \\
\text { - MDPt: } 139109 \$ \\
\text { - DC: } 142,053 \$ \\
\text { - DC and MD/DO: } \\
\text { 115,301\$ } \\
\text { - Sur/ED: } 87,661 \$\end{array}$ & \multirow[t]{3}{*}{$\begin{array}{l}\text { - MD/DO: } 135,824 \$ \\
\text { - MDPt: } 130,064 \$ \\
\text { - DC: } 132,989 \$ \\
\text { - DC and MD/DO: } \\
\text { 104,025\$ } \\
\text { - Sur/ED: } 60,807 \$\end{array}$} \\
\hline & & & & Health effects: & Adjusted costs: & \\
\hline & & & & $\begin{array}{l}\text { - - Saving in work } \\
\text { loss day in } \\
\text { comparison of } \\
\text { worker not } \\
\text { returning to work } \\
\text { (days } \mathrm{x} \text { wage) }\end{array}$ & $\begin{array}{l}\text { - MD/DO: } \\
\text { 11,289\$ } \\
\text { - MDPt: } 9046 \$ \\
\text { - DC: } 9065 \$ \\
\text { - DC and MD/DO: } \\
\text { 11277\$ } \\
\text { - Sx/ED: } 26,854 \$\end{array}$ & \\
\hline \multirow{7}{*}{$\begin{array}{l}\text { - Haas, } 2005[54] \\
\text { - US } \\
\text { - Cost-effectiveness } \\
\text { analysis/ prospective } \\
\text { cohort }\end{array}$} & \multirow{7}{*}{$\begin{array}{l}\text { - } 2780 \text { ambulatory adults } \\
\text { - LBP of mechanical origin } \\
\text { (acute and chronic } \\
\text { (7weeks) } \\
\text { - } 51 \text { chiropractic clinics and } \\
14 \text { general practice } \\
\text { community clinics in } \\
\text { Oregon and Washington }\end{array}$} & \multirow{7}{*}{$\begin{array}{l}\text { - Chiropractors } \\
\text { ( } n=1328 \\
\text { acute and } 527 \\
\text { chronic) } \\
\text { - Medical doctors } \\
\text { ( } n=615 \text { acute and } \\
310 \text { chronic) }\end{array}$} & \multirow{7}{*}{$\begin{array}{l}\text { - Healthcare } \\
\text { system } \\
\text { (Medicare)** } \\
\text { - } 12 \text { months } \\
\text { - Constant } 1995 \text { US } \\
\text { dollars }\end{array}$} & Costs: & $\begin{array}{l}\text { Adjusted mean } \\
\text { differences DC-MD } \\
\text { (SD) }\end{array}$ & $\begin{array}{l}\text { Cost-effectiveness } \\
\text { ratio }\end{array}$ \\
\hline & & & & \multirow{3}{*}{$\begin{array}{l}\text { - Office visit } \\
\text { - Radiograph } \\
\text { - Medication } \\
\text { - Advanced imaging } \\
\text { (imputed) } \\
\text { - Surgical consultation } \\
\text { (imputed) } \\
\text { - Physical therapists } \\
\text { referrals (imputed) }\end{array}$} & Acute patients & Acute patients \\
\hline & & & & & $\begin{array}{l}\text { - Pain: } 3.6(1.3) \\
\text { - Disability: } 2.7 \\
\quad(1.1) \\
\text { - Physical health: } \\
\text { 9.2(2.5) } \\
\text { - Mental health: } 5.4 \\
\quad \text { (2.5) } \\
\text { - Satisfaction: } 14.0 \\
\quad(3.1)\end{array}$ & \multirow[t]{2}{*}{$\begin{array}{l}\text { - Pain: } 17.6 \$ \\
\text { - Disability: } 23.6 \$ \\
\text { - Physical health: } \\
6.9 \$ \\
\text { - Mental health: } 11.7 \$ \\
\text { - Satisfaction: } 4.5 \$\end{array}$} \\
\hline & & & & & $\begin{array}{l}\text { Total cost: } 63 \$ \\
(69 \$)\end{array}$ & \\
\hline & & & & Health effects: & Chronic patients & Chronic patients \\
\hline & & & & \multirow[t]{2}{*}{$\begin{array}{l}\text { - Pain (100mm VAS) } \\
\text { - Functional status } \\
\text { (Oswestry } 100 \text { point } \\
\text { scale) } \\
\text { - Physical health } \\
\text { (SF-12) } \\
\text { - Mental health } \\
\text { (SF-12) } \\
\text { - Satisfaction (100 } \\
\text { point scale) }\end{array}$} & $\begin{array}{l}\text { - Pain: } 7.3(2.1) \\
\text { - Disability: } 5.4 \\
\quad(1.7) \\
\text { - Physical health: } \\
3.0(3.6) \\
\text { - Mental health: } 1.2 \\
\quad \text { (3.7) } \\
\text { - Satisfaction: } 18.1 \\
\text { (4.9) }\end{array}$ & \multirow[t]{2}{*}{$\begin{array}{l}\text { - Pain: } 0.1 \$ \\
\text { - Disability: } 0.1 \$ \\
\text { - Physical health: } \\
0.3 \$ \\
\text { - Mental health: } 1.0 \$ \\
\text { - Satisfaction: } 0.0 \$\end{array}$} \\
\hline & & & & & $\begin{array}{l}\text { Total cost: } 1.5 \$ \\
(117 \$)\end{array}$ & \\
\hline \multirow{4}{*}{$\begin{array}{l}\text { - Kominski, } 2005 \text { [56] } \\
\text { - US } \\
\text { - Cost minimisation } \\
\text { analysis/ randomized } \\
\text { controlled trial }\end{array}$} & \multirow{4}{*}{$\begin{array}{l}\text { - } 681 \text { adults members of } \\
\text { various HMOs } \\
\text { - LBP (with or without leg } \\
\text { symptoms) } \\
\text { - Large medical group } \\
\text { practice with } 3 \text { sites in } \\
\text { Southern California }\end{array}$} & \multirow{4}{*}{$\begin{array}{l}\text { - Medical care (MD) } \\
\text { ( } n=162) \text { Medical } \\
\text { care with physical } \\
\text { therapy (MDPt) } \\
\text { ( } n=167) \\
\text { - Chiropractic care } \\
\text { (DC)( } n=162) \\
\text { - Chiropractic care } \\
\text { with physical } \\
\text { modalities (DCPm) } \\
(n=163)\end{array}$} & \multirow{4}{*}{$\begin{array}{l}\text { - Healthcare } \\
\text { provider group ** } \\
\text { - } 18 \text { months } 1998 \\
\text { US dollars }\end{array}$} & Costs (charged): & Mean cost (SD): & \multirow[t]{4}{*}{ Not reported } \\
\hline & & & & $\begin{array}{l}\text { - Office visits } \\
\text { - Diagnostic services } \\
\text { - Therapeutic services }\end{array}$ & $\begin{array}{l}\text { - MD: } 647 \$(1755) \\
\text { - MDPt: } 1070 \$ \\
\text { (1454) } \\
\text { - DC: } 769 \$(1166) \\
\text { - DCPm: } 790 \$ \\
(765)\end{array}$ & \\
\hline & & & & Health effects: & $\begin{array}{l}\text { Mean Health } \\
\text { effect: }\end{array}$ & \\
\hline & & & & $\begin{array}{l}\text { - Pain Intensity } \\
\text { - Functional } \\
\text { status (Roland- } \\
\text { Morris) }\end{array}$ & $\begin{array}{l}\text { - No significant } \\
\text { differences } \\
\text { between groups }\end{array}$ & \\
\hline
\end{tabular}

* Cost of the original study were converted to 2015 US dollar using a web-based tool based PPP and GDPD values from the IMF[47]

** Precision obtained directly from the original author

doi:10.1371/journal.pone.0160037.t007 
Table 8. Quality assessment of economic evaluation of chiropractic cares for non-specific low back pain.

\begin{tabular}{|c|c|c|c|c|}
\hline \multicolumn{2}{|c|}{ Author (year) } & \multirow[t]{2}{*}{ Butler (2010) } & \multirow[t]{2}{*}{ Haas (2005) } & \multirow[t]{2}{*}{$\begin{array}{c}\text { Kominski } \\
(2005)\end{array}$} \\
\hline \multicolumn{2}{|c|}{ Study design } & & & \\
\hline 1 & $\begin{array}{l}\text { Was a well-defined question posed in answerable } \\
\text { form? }\end{array}$ & Yes & Not Clear & Yes \\
\hline 1.1 & $\begin{array}{l}\text { Did the study examine both costs and effect of the } \\
\text { service (s) or programme (s)? }\end{array}$ & Yes & Yes & No \\
\hline 1.2 & Did the study involve a comparison of alternatives? & Yes & Yes & Yes \\
\hline 1.3 & $\begin{array}{l}\text { Was a viewpoint for the analysis stated and was the } \\
\text { study placed in any particular decision-making } \\
\text { context? }\end{array}$ & Yes & No & No \\
\hline 2 & $\begin{array}{l}\text { Was a comprehensive description of the competing } \\
\text { alternative given? (that is, can you tell who did what } \\
\text { to whom, where, and how often?) }\end{array}$ & Yes & Yes & Yes \\
\hline 2.1 & Were any relevant alternatives omitted? & No & No & No \\
\hline 2.2 & $\begin{array}{l}\text { Was (Should) a do-nothing alternative (be) } \\
\text { considered? }\end{array}$ & $\begin{array}{l}\text { Not } \\
\text { appropriate }\end{array}$ & No & No \\
\hline 3 & $\begin{array}{l}\text { Was the effectiveness of the programme or } \\
\text { services established? }\end{array}$ & Yes & Yes & Yes \\
\hline 3.1 & $\begin{array}{l}\text { Was this done through a randomized, controlled clinical } \\
\text { trial? If so, did the trial protocol reflect what would } \\
\text { happen in regular practice? }\end{array}$ & No & No & Yes \\
\hline 3.2 & $\begin{array}{l}\text { Were effectiveness data collected and summarized } \\
\text { through a systematic review of studies? If so, were the } \\
\text { search strategy and rules for inclusion or exclusion } \\
\text { outlined? }\end{array}$ & No & No & No \\
\hline 3.3 & $\begin{array}{l}\text { Were observational data or assumptions used to } \\
\text { establish effectiveness? If so, what were the potential } \\
\text { biases in the results? }\end{array}$ & Yes & Yes & No \\
\hline 4 & $\begin{array}{l}\text { Were all the important and relevant costs and } \\
\text { consequences for each alternative identified? }\end{array}$ & Yes & Yes & No \\
\hline 4.1 & $\begin{array}{l}\text { Was the range wide enough for the research question } \\
\text { at hand? }\end{array}$ & Yes & Yes & Yes \\
\hline 4.2 & $\begin{array}{l}\text { Did it cover all relevant viewpoints? (Possible } \\
\text { viewpoints include the community or social viewpoints, } \\
\text { and those of patients and third-party payers. Other } \\
\text { viewpoints may also be relevant depending upon the } \\
\text { particular analysis.) }\end{array}$ & Yes & Yes & No \\
\hline 4.3 & $\begin{array}{l}\text { Were capital costs, as well as operating costs, } \\
\text { included? }\end{array}$ & No & No & No \\
\hline 5 & $\begin{array}{l}\text { Were costs and consequence measured accurately } \\
\text { in appropriate physical units (e.g. hours of nursing } \\
\text { time, number of physician visits, lost work days, } \\
\text { gained life-years)? }\end{array}$ & Yes & Yes & No \\
\hline 5.1 & $\begin{array}{l}\text { Were the sources of resources utilisation described } \\
\text { and justified? }\end{array}$ & Yes & Yes & No \\
\hline 5.2 & $\begin{array}{l}\text { Were any of the identified items omitted from } \\
\text { measurement? If so, does this mean that they carried } \\
\text { no weight in the subsequent analysis? }\end{array}$ & No/No & Yes/No & Yes/Yes \\
\hline 5.3 & $\begin{array}{l}\text { Were there any special circumstances (e.g. joint use of } \\
\text { resources) that made measurement difficult? Were } \\
\text { these circumstances handled appropriately? }\end{array}$ & Yes/Yes & Yes/Yes & Not clear \\
\hline 6 & Were costs and consequences valued credibly? & Yes & Yes & No \\
\hline
\end{tabular}


Table 8. (Continued)

\begin{tabular}{|c|c|c|c|c|}
\hline \multicolumn{2}{|c|}{ Author (year) } & \multirow[t]{2}{*}{ Butler (2010) } & \multirow[t]{2}{*}{ Haas (2005) } & \multirow[t]{2}{*}{$\begin{array}{c}\text { Kominski } \\
(2005)\end{array}$} \\
\hline \multicolumn{2}{|c|}{ Study design } & & & \\
\hline 6.1 & $\begin{array}{l}\text { Were the sources of all values clearly identified? } \\
\text { (Possible sources include market values, patient or } \\
\text { client preferences and views, policymakers' views and } \\
\text { health professionals' judgments.) }\end{array}$ & Yes & Yes & Yes \\
\hline 6.2 & $\begin{array}{l}\text { Were market values employed for changes involving } \\
\text { resources gained or depleted? }\end{array}$ & Yes & Yes & Yes \\
\hline 6.3 & $\begin{array}{l}\text { Were market values were absent (for example, } \\
\text { volunteer labour), or market values did not reflect } \\
\text { actual values (such as clinic space donated at a reduce } \\
\text { rate), were adjustments made to approximate market } \\
\text { values? }\end{array}$ & Yes & Yes & No \\
\hline 6.4 & $\begin{array}{l}\text { Was the valuation of consequences appropriate for the } \\
\text { question posed (that is, has the appropriate type of } \\
\text { analysis (CEA, CUA, CBA) been selected)? }\end{array}$ & Yes & Yes & No \\
\hline 7 & $\begin{array}{l}\text { Were costs and consequences adjusted for } \\
\text { differential timing? }\end{array}$ & $\begin{array}{c}\text { Not } \\
\text { appropriate }\end{array}$ & $\begin{array}{l}\text { Not } \\
\text { appropriate }\end{array}$ & $\begin{array}{l}\text { Not } \\
\text { appropriate }\end{array}$ \\
\hline 7.1 & $\begin{array}{l}\text { Were costs and consequences that occur in the future } \\
\text { "discounted" to their present values? }\end{array}$ & $\begin{array}{c}\text { Not } \\
\text { appropriate }\end{array}$ & $\begin{array}{c}\text { Not } \\
\text { appropriate }\end{array}$ & $\begin{array}{c}\text { Not } \\
\text { appropriate }\end{array}$ \\
\hline 7.2 & $\begin{array}{l}\text { Was any justification given for the discount rate(s) } \\
\text { used? }\end{array}$ & $\begin{array}{l}\text { Not } \\
\text { appropriate }\end{array}$ & $\begin{array}{c}\text { Not } \\
\text { appropriate }\end{array}$ & $\begin{array}{l}\text { Not } \\
\text { appropriate }\end{array}$ \\
\hline 8 & $\begin{array}{l}\text { Was an incremental analysis of costs and } \\
\text { consequences of alternative performed? }\end{array}$ & Yes & Yes & No \\
\hline 8.1 & $\begin{array}{l}\text { Were the additional (incremental) costs generated by } \\
\text { one alternative over another compared with the } \\
\text { additional effects, benefits, or utilities generated? }\end{array}$ & Yes & Yes & No \\
\hline 9 & $\begin{array}{l}\text { Was allowance made for uncertainty in the } \\
\text { estimation of costs and consequences? }\end{array}$ & No & Yes & Yes \\
\hline 9.1 & $\begin{array}{l}\text { If patient level data on cost or consequence were } \\
\text { available, were appropriate statistical analysis } \\
\text { performed? }\end{array}$ & Yes & Yes & Yes \\
\hline 9.2 & $\begin{array}{l}\text { If a sensitivity analysis was employed, was justification } \\
\text { provided for the ranges or distributions of values (for } \\
\text { key study parameters), and the form of sensitivity } \\
\text { analysis used? }\end{array}$ & No & No & No \\
\hline 9.3 & $\begin{array}{l}\text { Were the conclusions of the study sensitive to the } \\
\text { uncertainty in the results, as quantified by the statistical } \\
\text { and/or sensitivity analysis? }\end{array}$ & Yes & Yes & Yes \\
\hline 10 & $\begin{array}{l}\text { Did the presentation and discussion of the study } \\
\text { results include all issues of concern to user? }\end{array}$ & No & Yes & Yes \\
\hline 10.1 & $\begin{array}{l}\text { Were the conclusions of the analysis based on some } \\
\text { overall index or ratio of costs to consequences (e.g. } \\
\text { costs effectiveness ratio)? If so, was the index } \\
\text { interpreted intelligently or in a mechanistic fashion? }\end{array}$ & Yes & Yes & No \\
\hline 10.2 & $\begin{array}{l}\text { Were the results compared with those of other who } \\
\text { have investigated the same or similar questions? If so, } \\
\text { were allowances made for potential differences in study } \\
\text { methodology? }\end{array}$ & No & Yes & Yes \\
\hline 10.3 & $\begin{array}{l}\text { Did the study discuss the generalizability of the results } \\
\text { to other settings and patient/client groups? }\end{array}$ & No & Yes & Yes \\
\hline 10.4 & $\begin{array}{l}\text { Did the study allude to, or take account of, other } \\
\text { important factors in the choice or decision under } \\
\text { consideration (e.g., distribution of costs and } \\
\text { consequences, or relevant ethical issues)? }\end{array}$ & No & Yes & Yes \\
\hline
\end{tabular}

(Continued) 
Table 8. (Continued)

\begin{tabular}{|c|c|c|c|c|}
\hline \multicolumn{2}{|c|}{ Author (year) } & Butler (2010) & Haas (2005) & $\begin{array}{c}\text { Kominski } \\
(2005)\end{array}$ \\
\hline \multicolumn{5}{|c|}{ Study design } \\
\hline \multirow[t]{2}{*}{10.5} & $\begin{array}{l}\text { Did the study discuss issues of implementation, such } \\
\text { as the feasibility of adopting the preferred programme } \\
\text { given existing financial or other constraints, and } \\
\text { whether any freed resources could be redeployed to } \\
\text { other worthwhile programmes? }\end{array}$ & No & Yes & No \\
\hline & Overall quality assessment of the study & Low & High & Medium \\
\hline
\end{tabular}

doi:10.1371/journal.pone.0160037.t008

findings. Most notable was the study by Petersen et al., in which physical therapy care resulted in better global improvement at three months and higher functional status at 12 months, compared to chiropractic care in a population of subacute/chronic LBP [53]. When results were pooled with those from the other available studies for functional status at three [51] and 12 months [43, 44], the heterogeneity was considerable [59]. The inconsistent results can be explained by differences in settings (studies conducted in Denmark, UK or US); subpopulations (subacute/chronic vs. mixed symptom duration), and physical therapy approaches (McKenzie method vs. nonspecified physical therapy). We found one study, which favoured chiropractic care over physical therapy care in a mixed duration LBP population regarding global improvement at one month $[43,44]$. Finally, during our data extraction for the pooled effect estimates we found one outcome (the mental subscale of the SF-36 at the three months follow-up) that favoured chiropractic care over exercise therapy care in the study by Bronfort et al. [50]. The authors did not report this difference because they performed a multivariate analysis (instead of a final score comparison), which led them to report a non-significant difference.

The existing literature is currently too limited to allow for a synthesis of economic evaluations robust enough to offer guidance to decision makers, referring healthcare provider and patients about the cost-effectiveness of chiropractic compared to other types of care. Two out of three included economic evaluations had serious methodological limitations due to low sample size and omission of direct medical costs that could potentially impact the conclusions. Further, it is difficult to appreciate the robustness of the conclusions of the economic evaluations considering that sensitivity analyses were not reported.

\section{Consistency of findings with other studies and reviews}

Our results regarding the effectiveness of chiropractic care are consistent with previous reviews comparing chiropractors to other provider groups $[21,22]$ and SMT to other treatment modalities [24-26]. All have concluded that there is no clear superiority for any provider group or modality. Regarding economic evaluations, older reports [60, 61] have concluded that chiropractic care is highly cost-effective because of the relatively low consultation fee and the limited use of advanced diagnostic imaging. However, more recent rigorous systematic reviews of partial economic evaluations have failed to show an economic advantage of one type of care over another $[21,22]$. This is consistent with the findings from our review based on a limited number of full economic evaluations.

\section{Strengths and limitations of these reviews}

To our knowledge this is the first synthesis that exclusively considers pragmatic trials of chiropractic care without including fastidious trials of SMT. A comprehensive search was conducted to identify all relevant studies and a rigorous methodology was applied. The extraction process 
was performed in accordance with current guidelines and an experienced health economist supported the economic evaluations. Limiting our search to the past 25 years enabled us to select publications that were compatible with contemporary healthcare delivery. By only considering full economic evaluations and trials at a low risk of bias, we aimed to derive conclusions from more robust evidence.

Our review has limitations. First, we did not search the grey literature for clinical effectiveness studies. McAuley et al. showed that the inclusion of results from the grey literature tend to decrease effectiveness estimates in meta-analyses because the unpublished studies tend to report smaller treatment effects [62]. Second, critical appraisal requires scientific judgment that may vary among reviewers. This potential bias was minimized by training reviewers to use a standardized critical appraisal tool and using a consensus process among reviewers to reach decisions regarding scientific admissibility. Most of the original between-group differences and pooled estimates in our meta-analysis did not favour a specific provider group, and we believe it is unlikely that the inclusion of unpublished grey literature would change our conclusions. Third, the low number of clinical trials prevents us from conducting a meaningful investigation for publication bias. Fourth, the majority of the included clinical effectiveness studies (three out of five) and all three economic evaluations were conducted in the United States. Caution should therefore be used when generalizing our findings to other settings or jurisdictions. With respect to economic evaluations in particular, local healthcare systems and insurance plans may have a higher impact on cost than the type of healthcare provider [55].

\section{Recommendations for future researches}

Our search retrieved only a limited number of pragmatic clinical trials comparing chiropractic care to other types of care and only one high quality, full economic evaluation. We suggest therefore that future high quality pragmatic trials be conducted in parallel with full economic evaluations considering all relevant direct and indirect costs. The perspective of the economic evaluation should be clearly specified since it bears influence on the relevance of the health effects and cost considered. In addition, future studies should consider the impact of chiropractic care on return to work and include loss of income and wage compensation, a major component of indirect cost relevant to patients, the employers and society. Small differences in work absenteeism (indirect cost) have previously shown to have considerable impact on cost and the potential to reverse conclusions based on direct costs only [63, 64]. To facilitate the interpretation and decision-making process about types of care, we also suggest that future economic evaluations conduct willingness to pay and sensitivity analyses. Due to the large numbers of fastidious trials investigating the efficacy of SMT of LBP, researchers have previously called for a moratorium on future RCTs $[26,65]$. However, we believe that additional pragmatic, practice-based studies will help clarify whether chiropractic care is truly equivalent to other types of care in terms of effectiveness and cost. It is likely that, on average, there is not much difference between the different alternatives; however there may be heterogeneous treatment effects, i.e. some treatments that are cost-effective for some and not for others. Future studies could help elucidate this issue.

\section{Conclusion}

Moderate evidence suggests that chiropractic care for LBP appears to be equally effective as physical therapy. Limited evidence suggests the same conclusion when chiropractic care is compared to exercise therapy and medical care although no firm conclusion can be reached at this time. No serious adverse events were reported for any type of care. Our review was also unable to clarify whether chiropractic or medical care is more cost-effective. Given the limited 
available evidence, the decision to seek or to refer patients for chiropractic care should be based on patient preference and values. Future studies are likely to have an important impact on our estimates as these were based on only a few admissible studies.

\section{Supporting Information}

S1 Table. PRISMA Checklist.

(DOC)

\section{Acknowledgments}

The authors would like to thank Heather Owens for the linguistic revision on the manuscript.

\section{Author Contributions}

Conceived and designed the experiments: MAB AB MJS JB PH. Analyzed the data: MAB AB MJS RB. Contributed reagents/materials/analysis tools: MAB AB MJS JB PH RB. Wrote the paper: MAB AB MJS JB PH RB.

\section{References}

1. Deyo RA, Mirza SK, Martin BI. Back pain prevalence and visit rates: estimates from U.S. national surveys, 2002. Spine (Phila Pa 1976). 2006; 31(23):2724-7. Epub 2006/11/02. doi: 10.1097/01.brs. 0000244618.06877.cd 00007632-200611010-00014 [pii]. PMID: 17077742.

2. Leroux I, Dionne CE, Bourbonnais R, Brisson C. Prevalence of musculoskeletal pain and associated factors in the Quebec working population. Int Arch Occup Environ Health. 2005; 78(5):379-86. Epub 2005/04/22. doi: 10.1007/s00420-004-0578-2 PMID: 15843954.

3. Druss BG, Marcus SC, Olfson M, Pincus HA. The most expensive medical conditions in America. Health affairs (Project Hope). 2002; 21(4):105-11. Epub 2002/07/16. PMID: 12117121.

4. Asklof T, Martikainen J, Kautiainen H, Haanpaa M, Kiviranta I, Pohjolainen T. Paid expenditures and productivity costs associated with permanent disability pensions in patients with spinal disorders: Nationwide Finnish Register-based Study, 1990-2010. Eur Spine J. 2015. Epub 2015/01/31. doi: 10. 1007/s00586-015-3775-7 PMID: 25632839.

5. Vos T, Flaxman AD, Naghavi M, Lozano R, Michaud C, Ezzati M, et al. Years lived with disability (YLDs) for 1160 sequelae of 289 diseases and injuries 1990-2010: a systematic analysis for the Global Burden of Disease Study 2010. Lancet. 2012; 380(9859):2163-96. Epub 2012/12/19. S0140-6736(12) 61729-2 [pii] doi: 10.1016/S0140-6736(12)61729-2 PMID: 23245607.

6. Hoy D, March L, Brooks P, Blyth F, Woolf A, Bain C, et al. The global burden of low back pain: estimates from the Global Burden of Disease 2010 study. Annals of the Rheumatic Diseases. 2014; 73(6):96874. doi: 10.1136/annrheumdis-2013-204428 PMID: 24665116

7. Chou R, Qaseem A, Snow V, Casey D, Cross JT Jr., Shekelle P, et al. Diagnosis and treatment of low back pain: a joint clinical practice guideline from the American College of Physicians and the American Pain Society. Annals of internal medicine. 2007; 147(7):478-91. Epub 2007/10/03. 147/7/478 [pii]. PMID: 17909209.

8. Schmidt CO, Raspe H, Pfingsten M, Hasenbring M, Basler HD, Eich W, et al. Back pain in the German adult population: prevalence, severity, and sociodemographic correlates in a multiregional survey. Spine (Phila Pa 1976). 2007; 32(18):2005-11. Epub 2007/08/19. doi: 10.1097/BRS. Ob013e318133fad8 00007632-200708150-00014 [pii]. PMID: 17700449.

9. Cassidy JD, Carroll LJ, Côté P. The Saskatchewan Health and Back Pain Survey: The Prevalence of Low Back Pain and Related Disability in Saskatchewan Adults. Spine. 1998; 23(17):1860-6. PMID: 9762743

10. Foster NE, Hartvigsen J, Croft PR. Taking responsibility for the early assessment and treatment of patients with musculoskeletal pain: a review and critical analysis. Arthritis Res Ther. 2012; 14(1):205. Epub 2012/03/13. ar3743 [pii] doi: 10.1186/ar3743 PMID: 22404958; PubMed Central PMCID: PMC3392833.

11. Davis MA, Yakusheva O, Gottlieb DJ, Bynum JP. Regional Supply of Chiropractic Care and Visits to Primary Care Physicians for Back and Neck Pain. J Am Board Fam Med. 2015; 28(4):481-90. Epub 
2015/07/15. doi: 10.3122/jabfm.2015.04.150005 PMID: 26152439; PubMed Central PMCID: PMCPMC4512163.

12. Whedon JM, Song $Y$, Davis MA, Lurie JD. Use of chiropractic spinal manipulation in older adults is strongly correlated with supply. Spine (Phila Pa 1976). 2012; 37(20):1771-7. Epub 2012/04/11. doi: 10. 1097/BRS.0b013e31825762b7 PMID: 22487711; PubMed Central PMCID: PMCPMC3414681.

13. Whedon JM, Goertz CM, Lurie JD, Stason WB. Beyond spinal manipulation: should Medicare expand coverage for chiropractic services? A review and commentary on the challenges for policy makers. J Chiropr Humanit. 2013; 20(1):9-18. Epub 2014/07/30. doi: 10.1016/j.echu.2013.07.001 PMID: 25067927; PubMed Central PMCID: PMCPMC4111075.

14. Astin JA. Why patients use alternative medicine: results of a national study. Jama. 1998; 279 (19):1548-53. PMID: 9605899

15. Lonnberg F. [The management of back problems among the population. I. Contact patterns and therapeutic routines]. Ugeskr Laeger. 1997; 159(15):2207-14. Epub 1997/04/07. PMID: 9148544.

16. Wolsko PM, Eisenberg DM, Davis RB, Kessler R, Phillips RS. Patterns and perceptions of care for treatment of back and neck pain: results of a national survey. Spine (Phila Pa 1976). 2003; 28(3):2927; discussion 8. Epub 2003/02/05. doi: 10.1097/01.BRS.0000042225.88095.7C PMID: 12567035.

17. Kooijman M, Swinkels I, Leemrijse C, de Bakker D, Veenhof C. National Information Service of Allied Health Care. 2011.

18. Hurwitz EL, Chiang LM. A comparative analysis of chiropractic and general practitioner patients in North America: findings from the joint Canada/United States Survey of Health, 2002-03. BMC Health Serv Res. 2006; 6:49. Epub 2006/04/08. 1472-6963-6-49 [pii] doi: 10.1186/1472-6963-6-49 PMID: 16600038.

19. Cote $P$, Cassidy JD, Carroll L. The treatment of neck and low back pain: who seeks care? who goes where? Medical care. 2001; 39(9):956-67. Epub 2001/08/15. PMID: 11502953.

20. Kirby ER, Broom AF, Sibbritt DW, Refshauge KM, Adams J. Health care utilisation and out-of-pocket expenditure associated with back pain: a nationally representative survey of Australian women. PLoS One. 2013; 8(12):e83559. Epub 2014/01/01. doi: 10.1371/journal.pone.0083559 PMID: 24376716; PubMed Central PMCID: PMCPMC3869794.

21. Baldwin ML, Cote P, Frank JW, Johnson WG. Cost-effectiveness studies of medical and chiropractic care for occupational low back pain. a critical review of the literature. Spine J. 2001; 1(2):138-47. Epub 2003/11/01. S1529-9430(01)00016-X [pii]. PMID: 14588394.

22. Brown A, Angus $D$, Chen $S$, Tang Z, Milne S, Pfaff $J$, et al. Costs and outcomes of chiropractic treatment for low back pain (Structured abstract). Health Technology Assessment Database [Internet]. 2005; (2: ):[88 p.]. Available from: http://onlinelibrary. wiley.com/o/cochrane/clhta/articles/HTA32005000361/frame.html.

23. Gartlehner G, Hansen RA, Nissman D, Lohr KN, Carey TS. A simple and valid tool distinguished efficacy from effectiveness studies. Journal of clinical epidemiology. 2006; 59(10):1040-8. Epub 2006/09/ 19. doi: 10.1016/j.jclinepi.2006.01.011 PMID: 16980143.

24. Rubinstein SM, Terwee CB, Assendelft WJ, de Boer MR, van Tulder MW. Spinal manipulative therapy for acute low-back pain. Cochrane Database Syst Rev. 2012; 9:Cd008880. Epub 2012/09/14. doi: 10. 1002/14651858.CD008880.pub2 PMID: 22972127.

25. Rubinstein SM, van Middelkoop M, Assendelft WJ, de Boer MR, van Tulder MW. Spinal manipulative therapy for chronic low-back pain: an update of a Cochrane review. Spine (Phila Pa 1976). 2011; 36 (13):E825-46. Epub 2011/05/20. doi: 10.1097/BRS.0b013e3182197fe1 00007632-201106010-00016 [pii]. PMID: 21593658.

26. Rubinstein SM, Terwee CB, Assendelft WJ, de Boer MR, van Tulder MW. Spinal manipulative therapy for acute low back pain: an update of the cochrane review. Spine (Phila Pa 1976). 2013; 38(3):E15877. Epub 2012/11/22. doi: 10.1097/BRS.0b013e31827dd89d PMID: 23169072.

27. Shekelle PG, Adams AH, Chassin MR, Hurwitz EL, Brook RH. Spinal manipulation for low-back pain. Annals of internal medicine. 1992; 117(7):590-8. Epub 1992/10/01. PMID: 1388006.

28. Stano M, Haas M, Goldberg B, Traub PM, Nyiendo J. Chiropractic and medical care costs of low back care: results from a practice-based observational study. Am J Manag Care. 2002; 8(9):802-9. PMID: 12234021.

29. Walker BF, French SD, Grant W, Green S. A Cochrane review of combined chiropractic interventions for low-back pain. Spine (Phila Pa 1976). 2011; 36(3):230-42. Epub 2011/01/21. doi: 10.1097/BRS. Ob013e318202ac73 PMID: 21248591.

30. Busse JW, Jacobs C, Ngo T, Rodine R, Torrance D, Jim J, et al. Attitudes toward chiropractic: a survey of North American orthopedic surgeons. Spine (Phila Pa 1976). 2009; 34(25):2818-25. Epub 2009/11/ 17. doi: 10.1097/BRS.0b013e3181c1512f PMID: 19910864. 
31. Blanchette M-A, Rivard M, Dionne C, Cassidy JD. Chiropractors' characteristics associated with physician referrals: results from a survey of Canadian Chiropractors. J Manipulative Physiol Ther 2015; 38 (6):395-406. doi: 10.1016/j.jmpt.2014.11.001 PMID: 25939556

32. Nahal G, Hunnisett A, Cunliffe C. General practitioner referral for musculoskeletal therapies: Why physiotherapy and not chiropractic? Journal of Chiropractic Education. 2015; 29(1):82.

33. Dagenais S, Tricco AC, Haldeman S. Synthesis of recommendations for the assessment and management of low back pain from recent clinical practice guidelines. Spine J. 2010; 10(6):514-29. Epub 2010/ 05/25. S1529-9430(10)00288-3 [pii] doi: 10.1016/j.spinee.2010.03.032 PMID: 20494814.

34. Palmer S, Torgerson DJ. Economic notes: definitions of efficiency. Bmj. 1999; 318(7191):1136. Epub 1999/04/24. PMID: 10213735; PubMed Central PMCID: PMCPmc1115526.

35. Drummond MF, Sculpher M, Torrance G, O'Brien B, Stoddart G. Methods for the economic evaluation of health care programmes. New York: Oxford University Press 2005.

36. Briggs AH, O'Brien BJ. The death of cost-minimization analysis? Health economics. 2001; 10(2):17984. Epub 2001/03/17. doi: 10.1002/hec.584 PMID: 11252048.

37. Blanchette MA, Bussieres A, Stochkendahl MJ, Boruff J, Harrison P. Effectiveness and economic evaluation of chiropractic care for the treatment of low back pain: a systematic review protocol. Syst Rev. 2015; 4:30. Epub 2015/04/16. doi: 10.1186/s13643-015-0015-5 PMID: 25876025; PubMed Central PMCID: PMCPMC4369074.

38. Furlan $A D$, Pennick V, Bombardier $C$, van Tulder M. 2009 updated method guidelines for systematic reviews in the Cochrane Back Review Group. Spine (Phila Pa 1976). 2009; 34(18):1929-41. Epub 2009/08/15. doi: 10.1097/BRS.0b013e3181b1c99f PMID: 19680101.

39. Drummond MF, Sculpher MJ, Torrance GW, O'Brien BJ, Stoddart GL. Methods for the Economic Evaluation of Health Care Programmes 3ed. New York: Oxford University Press; 2005.

40. Tompa E, Culyer AJ, Dolinschi R. Economic Evaluation of Interventions for Occupational Health and Safety Developing Good Practice New York: Oxford University Press; 2008.

41. Drummond MF, Jefferson TO. Guidelines for authors and peer reviewers of economic submissions to the BMJ. The BMJ Economic Evaluation Working Party. BMJ. 1996; 313(7052):275-83. Epub 1996/08/ 03. PMID: 8704542; PubMed Central PMCID: PMC2351717.

42. group CBR. [cited 2014 June 20th]. Available from: http://back.cochrane.org/forms.

43. Meade TW, Dyer S, Browne W, Frank AO. Randomised comparison of chiropractic and hospital outpatient management for low back pain: results from extended follow up. Bmj. 1995; 311(7001):349-51. Epub 1995/08/05. PMID: 7640538; PubMed Central PMCID: PMCPmc2550426.

44. Meade TW, Dyer S, Browne W, Townsend J, Frank AO. Low back pain of mechanical origin: randomised comparison of chiropractic and hospital outpatient treatment. Bmj. 1990; 300(6737):1431-7. Epub 1990/06/02. PMID: 2143092; PubMed Central PMCID: PMCPmc1663160.

45. Hurwitz EL, Morgenstern H, Harber P, Kominski GF, Belin TR, Yu F, et al. A randomized trial of medical care with and without physical therapy and chiropractic care with and without physical modalities for patients with low back pain: 6-month follow-up outcomes from the UCLA low back pain study. Spine. 2002; 27(20):2193-204. PMID: 12394892.

46. Hurwitz EL, Morgenstern H, Kominski GF, Yu F, Chiang LM. A randomized trial of chiropractic and medical care for patients with low back pain: eighteen-month follow-up outcomes from the UCLA low back pain study. Spine. 2006; 31(6):611-21; discussion 22. PMID: 16540862.

47. Shemilt I, Thomas J, Morciano M. A web-based tool for adjusting costs to a specific target currency and price year. Evidence \& Policy: A Journal of Research, Debate and Practice. 2010; 6(1):51-9.

48. Slavin RE. Best evidence synthesis: an intelligent alternative to meta-analysis. Journal of clinical epidemiology. 1995; 48(1):9-18. Epub 1995/01/01. 0895-4356(94)00097-A [pii]. PMID: 7853053.

49. Moher D, Liberati A, Tetzlaff J, Altman DG. Preferred reporting items for systematic reviews and metaanalyses: the PRISMA statement. BMJ. 2009; 339:b2535. Epub 2009/07/23. PMID: 19622551; PubMed Central PMCID: PMC2714657. doi: 10.1136/bmj.b2535

50. Bronfort G, Maiers MJ, Evans RL, Schulz CA, Bracha Y, Svendsen KH, et al. Supervised exercise, spinal manipulation, and home exercise for chronic low back pain: A randomized clinical trial. Spine Journal. 2011; 11(7):585-98. http://dx.doi.org/10.1016/j.spinee.2011.01.036. PMID: 2011440834. doi: 10. 1016/j.spinee.2011.01.036

51. Cherkin DC, Deyo RA, Battie M, Street J, Barlow W. A comparison of physical therapy, chiropractic manipulation, and provision of an educational booklet for the treatment of patients with low back pain. $\mathrm{N}$ Engl J Med. 1998; 339(15):1021-9. PMID: 9761803.

52. Herzog W, Conway PJ, Willcox BJ. Effects of different treatment modalities on gait symmetry and clinical measures for sacroiliac joint patients. J Manipulative Physiol Ther. 1991; 14(2):104-9. PMID: 1826920. 
53. Petersen T, Larsen K, Nordsteen J, Olsen S, Fournier G, Jacobsen S. The McKenzie method compared with manipulation when used adjunctive to information and advice in low back pain patients presenting with centralization or peripheralization: a randomized controlled trial. Spine. 2011;36 (24):1999-2010. http://dx.doi.org/10.1097/BRS.0b013e318201ee8e. PMID: 21358492. doi: 10.1097/ BRS.0b013e318201ee8e

54. Haas M, Sharma R, Stano M. Cost-effectiveness of medical and chiropractic care for acute and chronic low back pain. J Manipulative Physiol Ther. 2005; 28(8):555-63. PMID: 16226622.

55. Butler RJ, Johnson WG. Adjusting rehabilitation costs and benefits for health capital: the case of low back occupational injuries. J Occup Rehabil. 2010; 20(1):90-103. http://dx.doi.org/10.1007/s10926009-9206-z. PMID: 19806437. doi: 10.1007/s10926-009-9206-z

56. Kominski GF, Heslin KC, Morgenstern H, Hurwitz EL, Harber PI. Economic evaluation of four treatments for low-back pain: results from a randomized controlled trial. Med Care. 2005; 43(5):428-35. PMID: 15838406.

57. Mosley CD, Cohen IG, Arnold RM. Cost-effectiveness of chiropractic care in a managed care setting. Am J Manag Care. 1996; 2:280-2.

58. Mushinski M. Treatment of back pain: outpatient service charges, 1993. Stat Bull Metrop Insur Co. 1995; 76:32-9.

59. Deeks JJ, Higgins J, Altman DG. Analysing Data and Undertaking Meta-Analyses. Cochrane Handbook for Systematic Reviews of Interventions: Cochrane Book Series. 2008:243-96.

60. Manga $P$, Angus DE. Enhanced chiropractic coverage under OHIP as a means of reducing health care costs, attaining better health outcomes and achieving equitable access to select health services: Faculty of Administration, University of Ottawa = Faculté d'administration, Université d'Ottawa; 1998.

61. Manga $P$, Angus D, Papadopoulos $C$, Swan W. The effectiveness and cost-effectiveness of chiropractic management of low-back pain: Pran Manga \& Associates Incorporated; 1993.

62. McAuley $L$, Pham $B$, Tugwell $P$, Moher $D$. Does the inclusion of grey literature influence estimates of intervention effectiveness reported in meta-analyses? Lancet. 2000; 356(9237):1228-31. Epub 2000/ 11/10. doi: 10.1016/s0140-6736(00)02786-0 PMID: 11072941.

63. Fayssoux R, Goldfarb NI, Vaccaro AR, Harrop J. Indirect costs associated with surgery for low back pain-a secondary analysis of clinical trial data. Popul Health Manag. 2010; 13(1):9-13. Epub 2010/02/ 18. doi: 10.1089/pop.2009.0061 PMID: 20158318.

64. Ivanova JI, Birnbaum HG, Kantor E, Schiller M, Swindle RW. Duloxetine use in employees with low back pain: treatment patterns and direct and indirect costs. Pain Med. 2014; 15(6):1015-26. Epub 2014/02/18. doi: 10.1111/pme.12362 PMID: 24529260.

65. Hurwitz EL. Commentary: Exercise and spinal manipulative therapy for chronic low back pain: time to call for a moratorium on future randomized trials? Spine J. 2011; 11(7):599-600. Epub 2011/08/09. doi: 10.1016/j.spinee.2011.04.021 PMID: 21821197. 